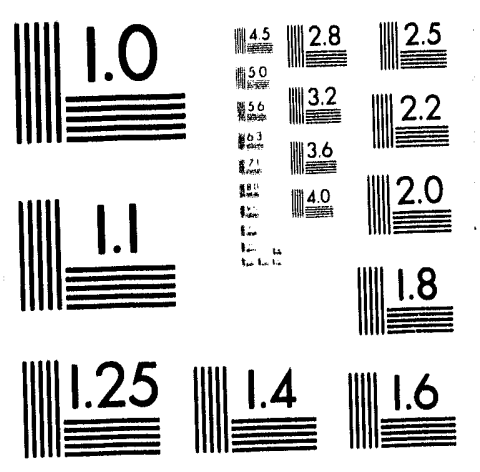



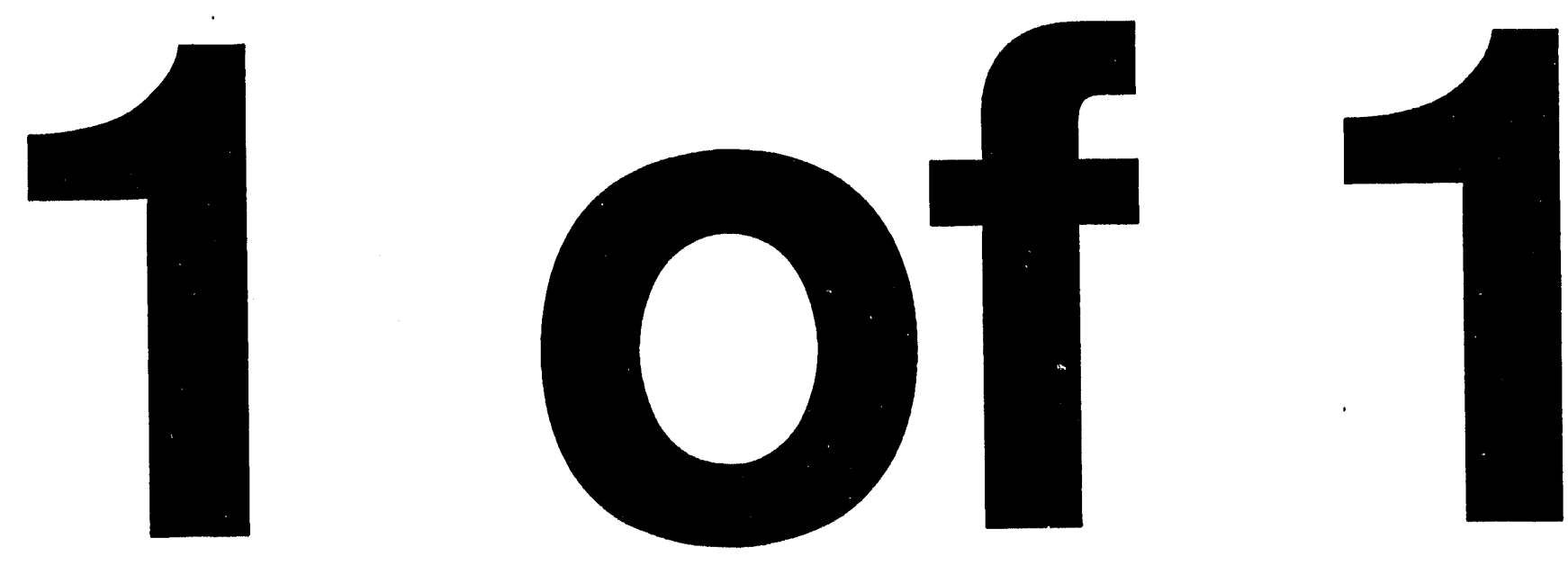


\title{
Parametric Instabilities in Picosecond Time Scales
}

\author{
H. A. Baldis \\ W. Rozmus \\ C. Labaune \\ Ph. Mounaix \\ D. Pesme \\ S. Baton \\ V. T. Tikhonchuk
}

This paper was prepared for submittal to the

NATO Advance Studies Institute

Cargese, Corsica, France

August 17-29, 1992

March 1, 1993

This is a preprint of a paper intended for publication in a journal or proceedings. Since changes may be made before publication, this preprint is made available with the understanding that it will not be cited or reproduced without the permission of the author.

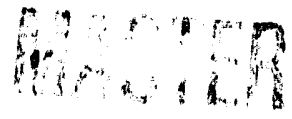




\section{DISCLAIMER}

This document was prepared as an account of work sponsored by an agency of the United States Government. Neither the United States Government nor the Uni versity of California nor any of their employees, makes any warranty, express or implied, or assumes any legal liability or responsibility for the accuracy, completeness, or usefulness of any information, apparatus, product, or process disclosed, or represents that its use would not infringe privately owned rights. Reference herein to any specific commercial products, process, or service by trade name, trademark, manufacturer, or otherwise, does not necessarily constitute or imply its endorsement, recommendation, or favoring by the United States Government or the University of California. The views and opinions of authors expressed herein do not necessarily state or reflect those of the United States Government or the University of Califomia, and shall not be used for advertising or product endorsement purposes. 


\title{
PARAMETRIC, INSTABIITTIES IN PICOSECOND TIME SCALES
}

\author{
H.A. Baldis \\ Lawrence Livermore National Laburatory, \\ l,ivermore, California 94550, USA \\ W. Rozmus \\ Department of Physics, University of Alberta, \\ Edmonton, Alberta, T6G 2J1, Canada. \\ C. Labaune, Ph. Mounaix, D. Pesme, S. Baton \\ Ecole Polytechnique \\ 91128 Palaiseau Cedex, Rrance
}

\author{
V.T. Tikhonchuk \\ P. N. Lebedev Physics Institute, \\ Russian Academy of Sciences, \\ Moscow, Russia
}

\section{INTRODUCTION}

The coupling of intense laser light with plasmas is a rich field of plasma physics, with many applications. Among these are inertial confinement fusion (ICF), $x$-ray lase:s, particle acceleration, and $x$-ray sources. Parametric instabilities have been studied for many years because of their importance to ICF; with laser pulses with duration of approximately a nanosecond, and laser intensities in the range $10^{14}-10^{15} \mathrm{~W} / \mathrm{cm}^{2}$ these instabilities are of crucial concern because of a number of detrimental effects. Although the laser pulse duration of interest for these studies are relatively long, it has been evident in the past years that to reach an understanding of these instabilities requires their characterization and analysis in picosecond time scales. At the laser intensities of interest, the growth rate for stimulated Brillouin scattering (SBS) is of the order of picoseconds, and of an order of magnitude shorter for stimulated Raman scattering (SRS).

In this paper we will discuss SBS and SRS in the context of their evolution in picosecond time scales. We will describe the fundamental concepts associated with their growth and saturation, and recent work on the nonlinear treatment required for the modeling of these instabilities at high laser intensities. Following this, we will review two types of 


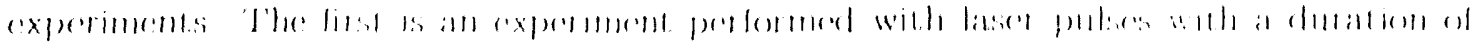

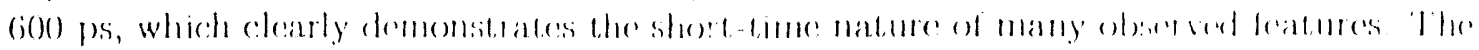

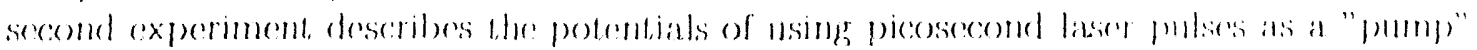

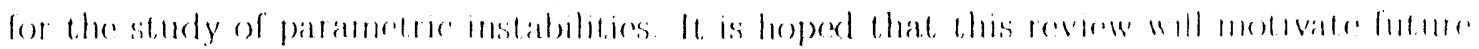

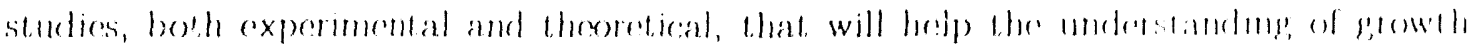
and saturation of enstabiluties and the interplay bow wern them.

\section{SCATTEIRING INSTABIIITIES IN UNDER-DENSE PIASMIAS}

The coupling between laser light and a plasma can be strongly influenered by the exen tation of waves by a varbinty of instabilities |1, 2|. These instabilities can be mest simply represented as the resonant coupling of the incident laser light into two enther waves. $\mathrm{Ne}$ glecting magnetic fields, these daughter waves are simply high frequency electron plasma waves (Langmuir), low frequency ion acoustic waves, and scattered electromagnetic waves. Two inportant instabilities are SRS and SBS. In SRS, the laser light can decay into a scattered light wave and an electron plasma wave. In the SBS instability, the two daughter waves are a scattered light wave and a low frequency ion acoustic wave Other important instability are the two plasmon decay instability in which the laser light drives a pair of electron plasma waves, and the decay into an electron plasma wave phus an ion acoustir wave (or a purely growing ion fluctuation). In addition to the above processes, the laser light can produce density modulations which lead to either self-focusimg or filamentation

\section{Stimulated Raman scattering}

The Raman instability (SRS) represents the resonant decay of an incident photon into a scattered photon plus an electron plasma wave $\{1,2,3 \mid$. The frequency and wave number matching conditions are

$$
\begin{aligned}
& \omega_{o}=\omega_{s}+\omega_{e p w} \\
& \mathbf{k}_{o}=\mathbf{k}_{s}+\mathbf{k}_{\text {epw }}
\end{aligned}
$$

where $\omega_{o}\left(\omega_{s}\right)$ and $k_{o}\left(k_{s}\right)$ are the frequency and wave number of the incident (scattered) light wave, and $\omega_{c p w},\left(k_{e p w}\right)$ is the frequency (wave number) of the electron plasma wave. Since $\omega_{s} \geq \omega_{e p w}$, this process requires that $\omega_{o}>2 \omega_{\text {epw }}$; i.e. $n_{e}<1 / 4 n_{c}$. The wavenumber $\mathrm{k}$ varies from $\mathrm{k}_{s} \sim \mathrm{k}_{o}$ for $n_{e} \sim n_{c} / 4$, to $\mathrm{k}_{s} \sim 2 \mathrm{k}_{o}$ for $n_{\mathrm{c}} \ll n_{c} / 4$. Similarly, the frequency of the backscattered Raman lighth varies from $\omega_{s} \sim 1 / 2 \omega_{o}$ at $n_{e} \sim n_{c} / 4$, to $\omega_{s} \sim \omega_{o}$ at $n_{e} \ll n_{c} / 4$. The frequency of the backscattered light is red shifted by the Bohm-Gross frequency $|1|$

$$
\omega_{B G}=\omega_{p} \sqrt{\left(1+\frac{12 \omega_{o}^{2}}{\omega_{p}^{2}} \frac{k T_{c}}{m c^{2}}\right)}
$$

where $\omega_{p}$ is the plasma frequency

$$
\omega_{p}=\sqrt{\frac{4 \pi n_{e}^{2}}{m_{e}}},
$$

$T_{e}$ is the electron temperature, $n_{e}$ is the electron density, and $n_{c}$ is the critical electron density.

To illustrate the physics of the Raman instability, consider a light wave with electric field amplitude $E_{o}$ and wavenumber $k_{o}$ propagating through a plasma. Iet the density be 


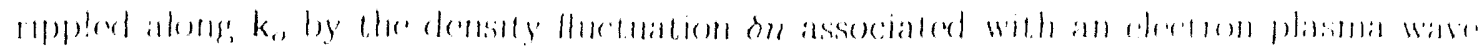

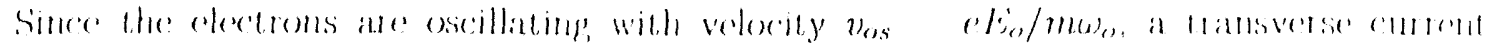

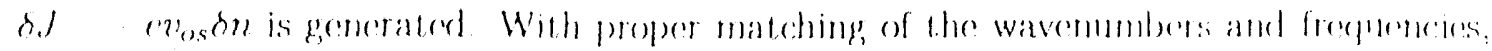

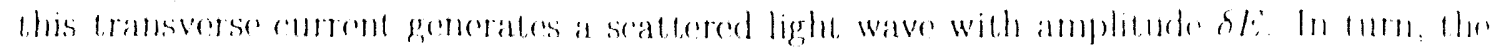
scatlered light wave beats with the incident light wave to produce a variaton in the wave pressure: $\nabla(6 \% / 8 \pi) \quad \nabla(6 \% \cdot \delta / \% / 4 \pi)$. The variation in the wave pressime pushes plasma from regions of high pressure to regions of low pressure, generatimg density perturbations: which can reinforce the initial fluctuation. The presence of this fredfack loop loads to the Raman instability.

Maximum growth oecurs when both the plasma wave and the down-shifterd lighte wave

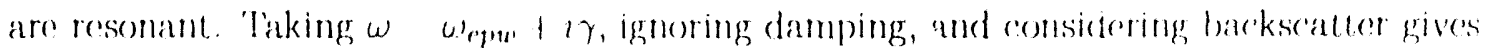

$$
\gamma \quad \frac{k v_{o s}}{4} \sqrt{\frac{\omega_{e p u}}{\omega_{o}-\omega_{e p u}}}
$$

where $\mathrm{k}$ is determined by the fresuency and wavemumber matching conditions

$$
\mathrm{k} \quad \mathrm{k}_{0}+\frac{\omega_{0}}{c} \sqrt{1-\left(\frac{\omega_{c p \omega}}{\omega_{o}}\right)^{2}}
$$

For example, the SRS growth rate for $0.35 \mu \mathrm{mm}$ light irradiating a plasma with a density of $0.2 n_{c}$ at an intensity of $10^{15} \mathrm{~W} / \mathrm{cm}^{2}$ is $\gamma \simeq 1.6 \times 10^{13} \mathrm{sec}^{-1}$, with a resulting growth time of approximately $0.07 \mathrm{ps}$.

Damping of both daughter waves introduces a pump threshold intensity for driving the instability. Damping can arise by both collisional and collisionless mechanisms and by inhomogeniety in the plasma. If we assume collisional damping of both the plasma wave and scattered light wave in an analogous fashion to inverse bremsstrahlung, net growth requires that

$$
\gamma>\frac{1}{2}\left(\frac{\omega_{c p w}}{\omega_{o}-\omega_{e p u}}\right) \nu_{e i}
$$

where ${ }^{\prime}{ }_{e i}$ is the electron-ion collision frequency. Collisional stabilization can be particularly effective in high $\mathrm{Z}$ plasmas irradiated by short wavelength laser light. For example, consider $0.35 \mu \mathrm{m}$ light incident on an Au plasma with an electron temperature of $2 \mathrm{keV}$, $\mathrm{Z}=50$, and density of $2 \times 10^{21} \mathrm{~cm}^{-3}\left(\sim n_{c}\right)$. Then $\nu_{c i} \sim 2.4 \times 10^{13} \mathrm{sec}^{-1}$ and the collisional threshold intensity would be about $5 \times 10^{14} \mathrm{~W} / \mathrm{cm}^{2}$.

A density gradient limits the region over which three waves can resonantly interact. Propagation of wave energy out of this region then represents an effective dissipation which must be overcome by the incident pump. To illustrate the gradient threshold, consider Raman backscatter at a density $n_{e}<<n_{c}$ in a plasma with a linear density profile with scale length, $L=|d / d x(\ln n)|^{-1}$. The Rosenbluth criterion for $e^{2 \pi}$ convective growth is

$$
\frac{\gamma^{2}}{\left|K v_{1} v_{2}\right|}>1
$$

where $\gamma$ is the growth rate in a uniform plasma, $v_{1,2}$ denotes the group velocity of the two daughter waves and $K$ is the gradient of the mismatch in the wavenumbers. Inserting the growth rate, we obtain

$$
\left(\frac{v_{o s}}{c}\right)^{2}>\frac{2}{k_{o} L}\left(\frac{c}{v_{g s}}\right) .
$$

$v_{g s}$ is the group velocity of the scattered light wave. For a plasma with $L \sim 10^{4} \lambda_{0}$ irradiated with $0.35 \mu \mathrm{m}$ laser light, this threshold intensity is only $3 \times 10^{14} \mathrm{~W} / \mathrm{cm}^{2}$. 
Stimulated Brillouin Soattoring

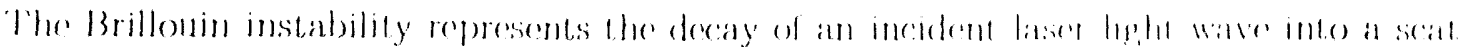
tered light wave plus an ion acoustic wave $|1,2,3|$ As can be reallly secon from the frepuency matching conditions, this instability is operative horoughom the plastna with $n<n_{c}$. In addition, by the Manley-Rowe relations, nearly all the onets. of the incedent

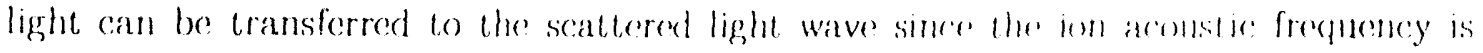

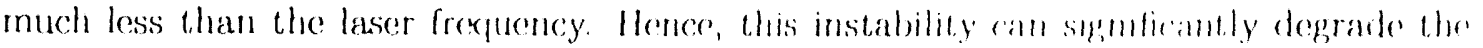
absorption and/or morlify its location!

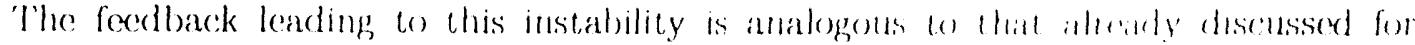
the Raman instability excepe now the density Huctuation is that atsinerated with an ion acoustic wave. Maximum growth occurs when the ion acollstire wave and down-shifted light wave are both resorant As an example, we consider backsiratlen ( $k$ 2 $2 k_{0}$ ) and neglect damping. For $\gamma \ll k c_{s}$ (weak coupling limit)

$$
\begin{aligned}
& \omega \quad k c s+i \gamma \\
& \gamma=\frac{1 k_{o} v_{o s} \omega_{m}}{2 \sqrt{2}} \text {. }
\end{aligned}
$$

For $0.35 \mu \mathrm{m}$ light with an intensity of $10^{14} \mathrm{~W} / \mathrm{cm}^{2}$ in a plasma with a density of $0.5 n_{c}$ and an electron temperature of $2 \mathrm{keV}, \gamma \sim 2.5 \times 10^{12}$ and the growth tume is about 0.4 ps. There is an instability threshold due to damping of the waves, so then net growth of Brillouin backscatter requires

$$
\frac{v_{o s}}{v_{c}}>4 \frac{\nu_{e i}}{\omega_{o}} \frac{\nu_{i}}{\omega_{i a}}
$$

where $v_{e}$ is the electron thermal velocity and $\nu_{e i}$ is the ion-electron collision frequency and $\nu_{i}$ is the ion damping rate. This intensity threshold is typically quite low.

Inhomogeneity in either density, expansion velocity, or temperature of the plasma introduces a threshold for the instability. Rosenbluth criterion for $e^{2 \pi}$ convective growth with. a gradient in expansion velocity gives for the backscatter instability

$$
\begin{aligned}
& \frac{v_{o s}}{v_{e}}>\frac{16}{k_{o} L_{\nu}} \frac{n_{c r}}{n} \\
& L_{\nu}=\left(\frac{1}{c_{s}} \frac{\delta v_{e}}{\delta x}\right)^{-1}
\end{aligned}
$$

If we consider a plasma with a density of $0.5 n_{c}$, an electron temperature of $2 \mathrm{keV}$, and $L_{\nu}=10^{3} \lambda_{o}$, the gradient threshold intensity is then about $10^{14} \mathrm{~W} / \mathrm{cm}^{2}$.

\section{SPATIAL AND TEMPORAL EVOLUTION OF PARAMETRIC INSTABIL- ITIES IN THE LINEAR APPROXIMATION}

In this section, we will restrict ourselves to static homogeneous plasmas and will consider 1D interacting plasme slab model in which $x=L$ and $x=0$ denote the points where the laser light, propagating from right to left, enters and goes out respectively. Such a 1D modelization is considered to describe properly the backscattering instabilities (beam to beam reflection). 
Standard Threer Wase ('omplings

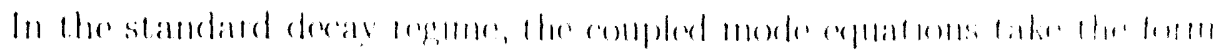

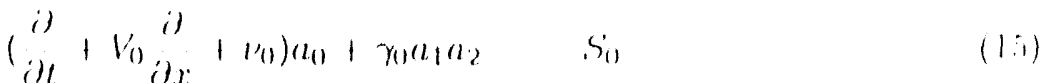

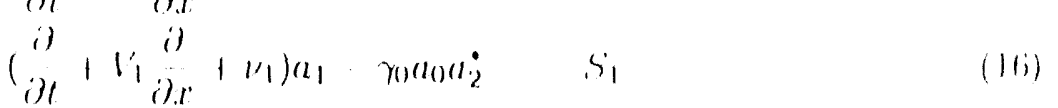

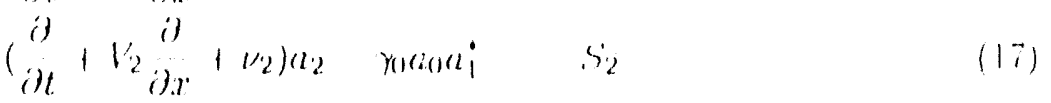

where $a_{0}, a_{1}$ and $a_{2}$ stand for the amplitudes of the incoming laser light, the transverse and the longrtuditial danghter waves respertively. These amplitudes have hern normalized on have $a_{0}(L) \quad 1$ at the incoming point $x$ L and at the intensily peak of the laser irradiation; $\gamma_{0}$ is the linear homogenesus growth rate of the paratnetric instability. The quantities $V_{\alpha}$ and $\nu_{\alpha}$ denote the group velocity and the linear damping of wave $\alpha$. In this section we will restrict ourselves to backscattering instabilities only, so that ond has $V_{0}<0, V_{1}>0$ and $V_{2}<0$. The source terms $S_{a}$ on the right-hand side of lags. (15i), (16), (17) account for the thermal noise emission of wave a and are therefore stochastic functions in space and time. The statistical properties of the source functions $S_{\alpha}$ will be described in the next Subsection.

Equations $(15,16,17)$ are correct only in the so-called standard dexay limit where the envelope approximation is valid for each of the interacting waves. In the case of SRS the validity condition for this approximation can be written in physical units as

$$
I_{14} \lambda^{2}<<3.6410^{5} \frac{\left(n / n_{c}\right)^{1 / 2}\left|1-\left(n / n_{c}\right)^{1 / 2}\right|}{\left|\sqrt{1-n / n_{c}}+\sqrt{1-2\left(n / n_{c}\right)^{1 / 2}}\right|^{2}}
$$

and in the case of SBS it reads

$$
I_{14} \lambda^{2}<<1.5 \frac{n_{c}}{n} \sqrt{1-n / n_{c}}(Z / A)^{1 / 2}\left|T_{e}+\frac{3 T_{i}}{Z}\left(1+K_{2}^{2} \lambda_{D}^{2}\right)\right|^{3 / 2} \sqrt{1+K_{2}^{2} \lambda_{D}^{2}}
$$

where $T_{e}$ and $T_{i}$ are the electron and ion temperature in units of $k e V_{i}$ the quantity $K_{2}^{2} \lambda_{D}^{2}$ is given by $K_{2}^{2} \lambda_{D}^{2}=7.8310^{-3} T_{e}\left(n_{c} / n-1\right)$. In both expressions $(18,19) I_{14} \lambda^{2}$ denotes the laser flux in units of $10^{14} \mathrm{~W} \mathrm{~cm}^{-2}^{2} \mathrm{~m}^{2}$.

\section{Statistical Properties of the Source Terms $S_{\alpha}$}

The source terms $S_{\alpha}$ on the right-hand side of Eqs. $(15,16,17)$ are stochastic functions in space and time which have to reproduce properly the equilibrium statistical properties of fields $a_{\alpha}$ within the spectral domain of interest for the instability. These stochastic processes will have the form of white noises with the following statistical properties

$$
\begin{gathered}
\left\langle S_{\alpha}(x, t)\right\rangle=0 \\
\left\langle S_{\alpha}(x, t) S_{\alpha}^{*}\left(x^{\prime}, t^{\prime}\right)\right\rangle=(2 \pi)^{2} \Sigma_{\alpha}^{1 D} \delta\left(x-x^{\prime}\right) \delta\left(t-t^{\prime}\right)
\end{gathered}
$$

where \langle\rangle denotes statistical average. The space-time variables appearing in Eqs. $(20,21)$ are the slowly varying quantities corresponding to the envelope approximation. The II) spectral density $\Sigma_{\alpha}^{1 D}$ is determined from the condition that the equilibrium fuctuations 
(a) 10 .

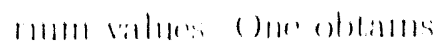

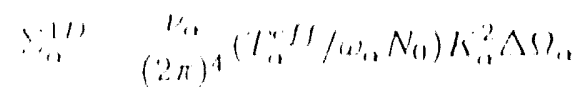

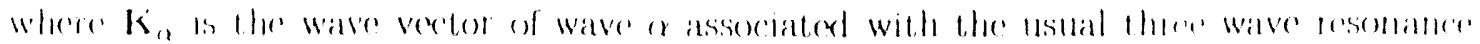

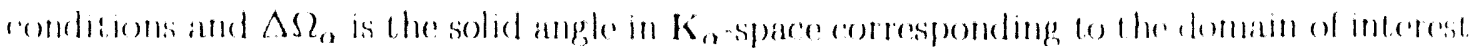

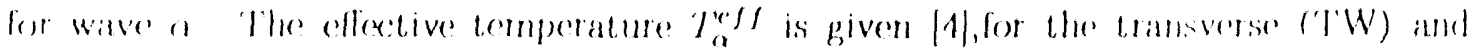

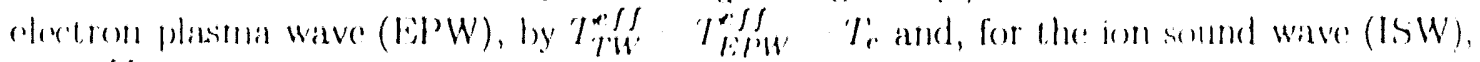

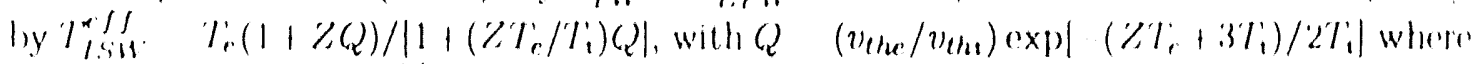

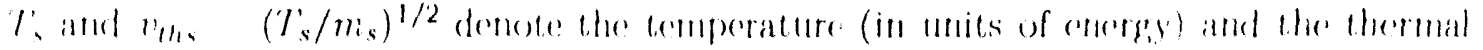

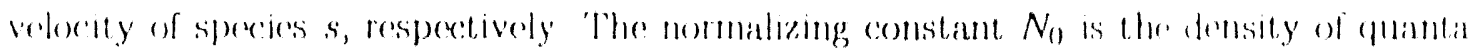
forl llor plump wave

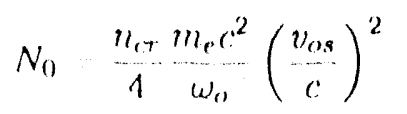

where $v_{o s}$ is the electron quiver velocity $v_{0 s} q_{r} E_{0} / l_{0} w_{0}$, tio denoting the maximmm amplitude of the pump wave electric field at its entry point $x=L$. In the case of eomparison with ID) particle in cell (PIC) simulations, the whole factor $T_{1}^{\prime} K_{2}^{2} \Delta s_{\alpha}$ should be determined to match the equilibrium characteristics of the mumerical scheme.

\section{Space-Time Behavior in the Linear Regime}

In the limit of small amplitude daughter waves, i.e. for $\left|a_{1}\right|<<1$ and $\left|a_{2}\right|<<1$, once cant neglect the nonlinear pump depletion and solve the linearized system of liss. $(15,16,17)$ where $a_{0}$ is now a given function of space and time. In the case of a rarefied $\left(n / n_{c}<<1\right)$ and optically thin $\left(\nu_{0} L /\left|V_{0}\right|<<1\right)$ interacting plasma one can also neglect the lincar dispersion and damping of the incident pump wave so that the pulse shape remains constant during a propagation through the interacting plasma. For the sake of simplicity we model the incident pulse of peak intensity $I_{\max }$ and characteristic duration $t_{p}$ by a square pulse of intensity $I_{0}$ and duration $t_{p}$. The intensity $I_{0}$ is computed from the condition that the energy of the square pulse be identical to the incident energy. Accordingly, $a_{0}$ is equal to unity within the pulse interval $\max |0, L-| V_{0}|t| \leq x \leq \min |L, L,-| V_{0}^{\prime}\left|\left(t-t_{p}\right)\right|$ and $t_{0}$ zero elsewhere. This simple modeling is justified by numerical solutions to Was.(1) which show that varying the pulse shape does not lead to significantly different values of the time integrated reflectivity |5|. The solution of the system (16)-(17) with a square pulse as a pump wave can be carried out by means of the Green's function method after specifying, the initial and boundary conditions $|6|$. For the initial stage of the pulse front propagation through the plasma, i.e. for $t<L /\left|V_{0}\right|$, we can assume vanishing initial conditions for the plasma fluctuations. Instead, we can impose the boundary conditions at the front of the pulse corresponding to the thermal equilibrium, because the laser pulse encounters a thermal plasma at its front. In the subsequent period of time $t>L /\left|V_{0}\right|$, one has to solve the system (16)-(17) taking for $a_{\alpha}(x, t)$ at $t=L /\left|V_{0}^{\prime}\right|$ the value reached at the end of the initial period and imposing thermal equilibrium for $a_{1}$ at $x \ldots 0$. One can neglect the additional boundary condition corresponding to thermal equilibrium for an at $x \quad l$ since, in the domain of parameters of concern in a picosecond time scale laser-plasma interaction, it would not lead to significantly different results from those obtained without it except within a negligibly thin boundary layer located around $x=1$. Following the procedure described above one obtains the fluctuations $\left\langle\left|a_{\alpha}\right|^{2}\right\rangle$ as complicated integrals over space and time which can be calculated either asymptotically in the limit $\gamma_{0} \rightarrow \infty$ (large gain factor limit) or perturbatively in the limit $\gamma_{0} \rightarrow 0$ (small gain factor limit). From these two limits it is possible to design $|6|$ tractable and uniform approximations to the fuctuations $\left\langle\left|a_{\alpha}\right|^{2}\right\rangle$, i.e. expressions which are correct for both small and large values 


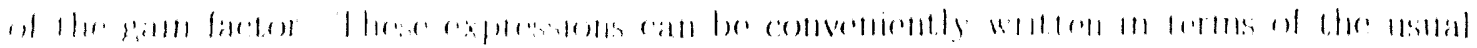

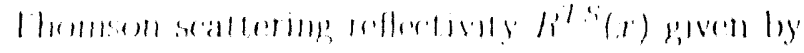

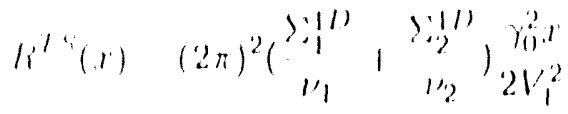

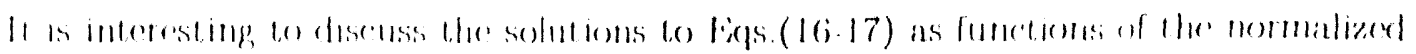

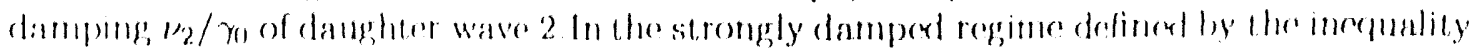
$1.2 / 3) \cdot\left(1\left|V_{1} /\right| V_{0} \mid\right)^{1 / 2}$, ono obtams the following expressions

$$
\begin{aligned}
& \left\langle\left|a_{1}(x, 1)\right|^{2}\right\rangle \quad\left\langle\left|n_{1}\right|^{2}\right\rangle_{n, n} \mid
\end{aligned}
$$

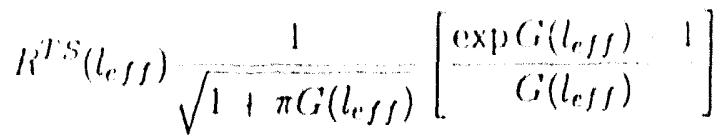

$$
\begin{aligned}
& \left\langle\left|a_{2}(r, t)\right|^{2}\right\rangle \quad\left\langle\left|a_{2}\right|^{2}\right\rangle_{e q} \mid
\end{aligned}
$$

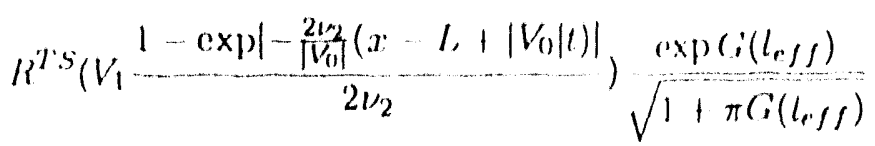

where $\left\langle\left|a_{\alpha}\right|^{2}\right\rangle_{\text {ey }}$ denotes the equilibrium fluctuations of wave a corresponding to the limit $\gamma_{0}$ o The gain factor $G(x)$ and the effective length $l_{e} s$ are given by

$$
\begin{array}{ll}
G(x) & =\frac{2 \gamma_{0}^{2} x}{\nu_{2} V_{1}} \\
l_{e J 1} & =\operatorname{Min}\left|x, \frac{V_{1}\left(x+1, V_{0}(t)\right)}{\left|W_{0}\right|+V_{1}}\right| .
\end{array}
$$

From the expression of the gain factor $G\left(l_{e f f}\right)$ appearing in Exps. $(26,27)$, one can see that the interaction is characterized by two different phases. The first phase takes place in the time interval $(L-x) /\left|V_{0}\right|<t<L /\left|V_{0}\right|+x / V_{1}$ and corresponds to exponentially growing in time fluctuations (and logarithmic corrections). The wave amplitude instantaneous growth rate $\gamma \equiv \gamma_{0}^{2} /\left|\nu_{2}\left(1+V_{1} /\left|V_{0}\right|\right)\right|$ may be interpreted as corresponding to a spatial amplification in the frame moving with the laser front. One has $G\left(l_{e f f}\right)=2 K X(t)$ where $K=\gamma_{0}^{2} /\left|\nu_{2}\left(V_{1}+\left|V_{0}\right|\right)\right|$ denotes the spatial amplification growth rate in the inoving frame, and where $X(t)=x-L+\left|V_{0}\right| t$ is the instantaneous distance between the pulse front and the point $x$. The second phase takes place in the time interval $L /\left|V_{0}\right|+x / V_{1}<t<$ $t_{p}+(L-x) /\left|V_{0}\right|$ and corresponds to the spatial amplification in the laboratory frame. During this phase the gain factor $G\left(l_{c / f}\right)$ remains equal to the usual spatial amplification factor $G\left(l_{e f f}\right)=\left(2 \gamma_{0}^{2} x\right) /\left(\nu_{2} V_{1}\right)$ and the fluctuations $\left\langle\left|a_{\alpha}\right|^{2}\right\rangle$ do not longer evolve in time. In the complementary, weakly damped regime defined by the inequality $\nu_{2} / \gamma_{0} \ll(1)$ $\left.V_{1} /\left|V_{0}\right|\right)^{-1 / 2}$, one obtains the expressions

$$
\begin{aligned}
& \left\langle\left|a_{1}(x, t)\right|^{2}\right\rangle=\left\langle\left|a_{1}\right|^{2}\right\rangle_{e q} t \\
& R^{T S}\left(l_{e / \int}\right) \frac{\exp \Gamma\left(\tau_{e / 1}\right)}{\sqrt{11+q_{1}^{2} G\left(\lambda_{e / f}\right) \| 1+r_{1}^{2} G\left(\lambda_{e / f}\right)_{1}^{\prime}}}\left[\frac{\exp G\left(\lambda_{e / 1}\right)-1}{G\left(\lambda_{e j}\right)}\right] \\
& \left\langle\left|a_{2}(x, t)\right|^{2}\right\rangle=\left\langle\left|a_{2}\right|^{2}\right\rangle_{e q}+R^{T S}\left(\frac{v_{1}\left(z-t+1 v_{0} \mid t\right)}{\left|v_{0}\right|}\right) \\
& {\left[\frac{\exp G\left(\lambda_{e f f}\right)-1}{G\left(\lambda_{e f f}\right)}\right] \frac{\exp \Gamma\left(r_{e f}\right)}{\sqrt{\left|1+q_{2}^{2} G\left(\lambda_{e f f}\right)\right|\left|1+r_{2}^{2} G\left(\lambda_{e f f}\right)\right|}}}
\end{aligned}
$$




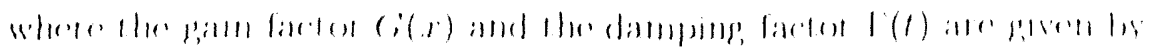

$$
\begin{aligned}
& \text { (in) } \quad 2 \pi x \\
& \text { l'(1) } \quad 21 \% 2
\end{aligned}
$$

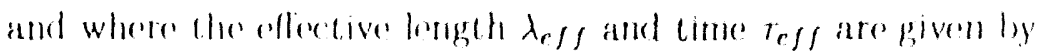

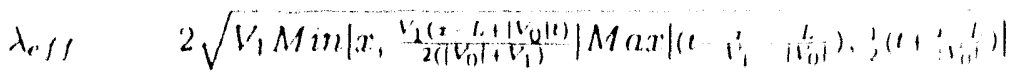

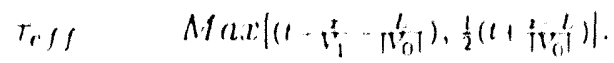

Whe quanticies qo and $r_{a}$ aro defined by

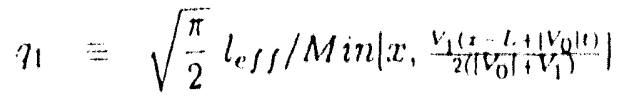

$$
\begin{aligned}
& \eta_{2} \equiv \sqrt{\frac{\pi}{2}}\left(t+\frac{x-L}{\left|V_{0}\right|}\right) / M a x \mid\left(t-\frac{x}{v_{1}}-\mid \frac{t}{\mid V_{0}}\right), \frac{1}{2}\left(t+\mid v_{0} t\right)
\end{aligned}
$$

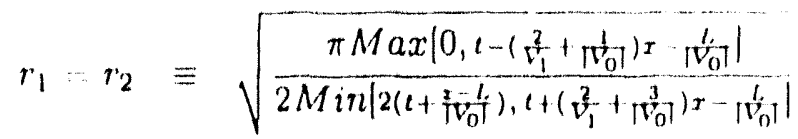

The validity domain of the expressions (29), (30) is actually restricted by the inequality $t<t^{*}$, where $t^{*} \sim \operatorname{Min}|L /| V_{2}\left|,\left(\gamma_{0}^{2} L\right) /\left(\nu_{2}^{2} V_{1}\right)\right|$ is the characteristic timo at which the fluctuations behave as predicted by the usual time asymptotic analysis (namely, they reach steady state in the convective regime or grow in time in the absolute regimo). $I t$ is important to realize that it takes the time $t^{*}$ to distinguish betwon absolute and convective evolution. In the case of short pulse experiments in prefonned plasma, the pulse duration $t_{p}$ is typically much shorter than $t^{*}$ so that the concepts of absolute or convective instabilities are irrelevant for the proper description of the scattering instabilities $|7,8|$. In this case, the whole interaction takes place in the transient stage of the evolution which is correctly given by the Eqs.(29), (30). From the expression of the gain factor $G\left(\lambda_{e f f}\right)$ appearing in Eqs. (29), (30), one finds that the transient stage $\left(t<t^{*}\right)$ of the interaction is characterized by two different regimes. The first phase takes place in the time interval ( $L$... $x) /\left|V_{0}\right|<t<L /\left|V_{0}\right|+\left(x / V_{1}\right)\left(2+V_{1} /\left|V_{0}\right|\right)$ and corresponds to exponentially growing in time fluctuations (and logarithmic corrections). The wave amplitude instantaneous growth rato $\gamma \equiv \gamma_{0} /\left(1+V_{1} /\left|V_{0}\right|\right)^{1 / 2}$ can again be interpreted as corresponding to a spatial amplification in the frame moving with the pulse front. One indeed finds that $G\left(\lambda_{e f}\right)=2 K X(t)$, where the spatial amplification growth rate $K$ in the moving frame is now computed in the weakly damped limit, namely $K=\gamma_{0} / \sqrt{\left(V_{1}+\left|V_{0}\right|\right)\left(V_{2}+\left|V_{0}\right|\right)} \simeq \gamma_{0} /\left|V_{0}\right| \sqrt{1+V_{1} /\left|V_{0}\right|}$. One should stress that neglecting the pulse propagation amounts to taking $\left|V_{0}\right|=+\infty$, which would lead to overestimate of the instantaneus growth rate by the factor $\sqrt{2}$ in the underdense limit $\left|V_{0}\right| \sim\left|V_{1}\right| \sim c$. The second phase of the evolution takes place in the subsequent time interval $L /\left|V_{0}\right|+\left(x / V_{1}\right)\left(2+V_{1} /\left|V_{0}\right|\right)<t<t_{p}+(L-x) /\left|V_{0}\right|$ and is associated with fluctuations behaving like $\exp G_{\text {tot }}$ (plus logarithmic corrections), where the total gain factor $G_{t o t} \equiv G\left(\lambda_{e f f}\right)+\Gamma\left(\tau_{e f f}\right)$ is given by

$$
G_{\text {tot }}=4 \gamma_{0} \sqrt{\frac{x}{V_{1}}\left(t-\frac{x}{V_{1}}-\frac{L}{\left|V_{0}\right|}\right)}-2 \nu_{2}\left(t-\frac{x}{V_{1}}-\frac{L}{\left|V_{0}\right|}\right)
$$

which corresponds to a time behavior which is not of an exponential type $|6,7,9|$. The effective wave amplitude instantaneous growth rate $\gamma_{e f f}(t)$ defined by $\gamma_{e} f(t) \equiv \frac{1}{\left|a_{o}\right|} \frac{\partial\left|a_{a}\right|}{\delta t}$ is a decreasing function of time which reads 


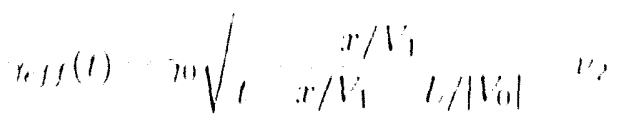

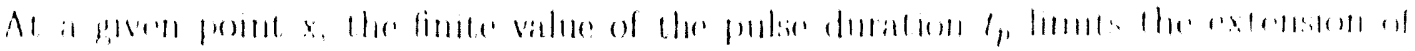

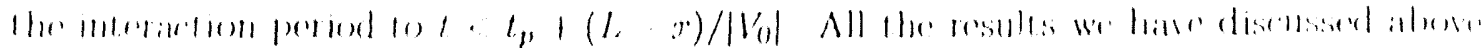

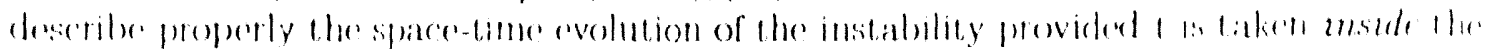

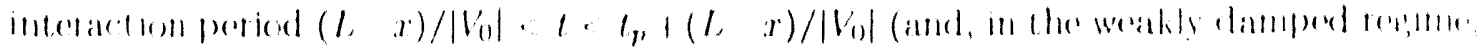

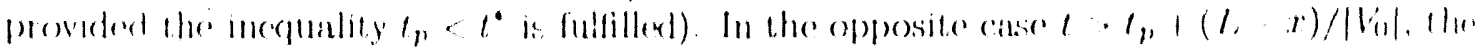

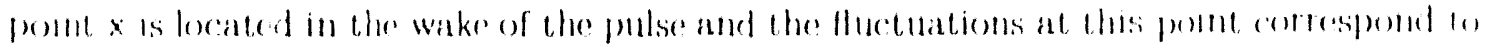

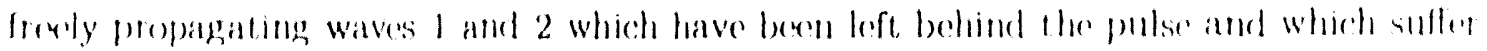

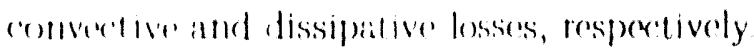

\section{NONLINEAR EVOLUTION OF STIMULATED RAMAN S(:ATTERING:}

In our revew of scattering instabilities on the short time scale we discuss physical pro cesses which ato not aftected by the hydrodymamic ovolution of laser produced plasmas Our simplified theoretical models treat plasma as a homogeneous mexhum vith the fixerl donsity and temperatures, but include soveral andincar tems describing enmpleatcul wave rvolution. It has become apparent that the proper understanding of expretimental results must involve nonlinear physies even on the scale of several picoseconds liast growing SRS can easily reach nonlinear regime within this time period. The primary nonlinear effects related to SRS correspond to coupling between langmuir and ion acoustic: waves, which results in parametric decay cascade and/or langmuir collapse. The broad spectra of langmuir and jon acoustic fluctuations produced during this nomlinear SRS evolution can have in turn dramatic effect on other, slowly growing scatlering processes including; Brillouin, forward Raman and non resonant interactions involving anti-Stokes components of electromagnetic waves. Review of recent theoretical results on the saturation of SRS and the effect of SRS produced non thermal fluctuation levels on the secondary seattering processes will be given in the next two subsections.

\section{Theoretical Model}

Since $1987(9,10,11)$ the nonlinear evolution of SRS has been studiex within the context of the model, which includes the system of Zakharov and electromagnetic wave equations. The Zakharov equations describe coupling between Langmuir and sound waves. They include physics related to modulational instability, parametric decay instability (PDI), PDI cascade, and the effects related to strong Langmuir turbulence and collapse. The ponderomotive force of electromagnetic waves drives the SRS resonant Langmuir wave at the relatively large wave number, making a modulational instability the unlikely mechanism of nonlinear SRS saturation (11). An important nonlinear coupling describer by this model $\{9,10,11\}$ corresponds to interaction between stimulated Brillouin scattering and SRS. SBS produces sound waves, which can affect nonlinear evolution of Langmuir waves by destroying SRS matching conditions or by triggering the localization of langmuir fields. The actual scenario of SRS/SBS coupling depends on the linear growth of both instabilities and initial noise levels of plasma fluctuations (cf.previous section). We have identified the range of plasina parameters, relevant for many current short wavelength laser-plasma interaction experiments, where SBS does not grow in time and has negligible effect on the evolution of SRS at early times. One can achieve similar conditions for even wider range of parameters but for the short laser pulses, when slowly growing SBS has no time to reach large levels of reflectivity during the laser pulse duration. By eliminating at first strong SBS we are able to make progress in our understanding of nonlinear SRS behavior. The 


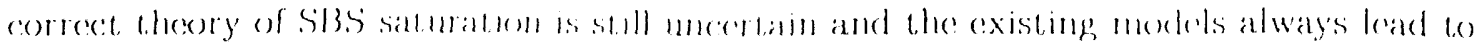
much higher values of reffectivity as compared to experimental observations. Next seetion will address some of the guestions retated to the saturation of SBS in the context of 21 ) modeling of the instability linfortmately analysis of the two dimensiomal models of SRSS is still impractical from the theoretical and mmmerical point of view. ()ur conchusions are based on the mumerical solutions to the system of Zakharov and elertromagnetic wave equations

$$
\begin{aligned}
& i\left(\frac{\partial E}{\partial t}+\omega_{t} \cdot E\right)+\frac{3 v_{c}^{2}}{2 \omega_{p}} \frac{\partial^{2} E}{\partial x^{2}}-\frac{\omega_{p}}{2} N E \\
& =\frac{\pi e n_{0}}{\omega_{p}} \frac{\partial}{\partial x}\left(\Psi_{0} \Psi_{i}^{*}\right)+S_{L}(x, t) \\
& \frac{\partial^{2} N}{\partial t^{2}}+2 \nu_{A} \cdot \frac{\partial N}{\partial t}-c^{2} \frac{\partial^{2} N}{\partial x^{2}} \\
& =\frac{\partial^{2}}{\partial x^{2}}\left(\frac{|E|^{2}}{16 \pi n_{0}^{i} m_{\boldsymbol{i}}}+\frac{Z m_{e}}{4 m_{\boldsymbol{i}}}\left|\Psi_{0}\right|^{2}\right)+S_{A}(x, t), \\
& i\left(-\frac{\partial \Psi_{0}}{\partial t}+\nu_{0} \Psi_{0}^{\prime}\right)+\frac{c^{2}}{2 \omega_{0}} \frac{\partial^{2} \Psi_{0}^{\prime}}{\partial x^{2}}-\frac{\omega_{p}^{2}-\omega_{0}^{2}}{2 \omega_{0}} \Psi_{0} \\
& =\frac{\omega_{p}^{2}}{2 \omega_{0}} N \Psi_{0}-\frac{e}{4 \omega_{0} m_{e}}\left(\Psi_{1} \frac{\partial E}{\partial x}\right), \\
& i\left(\frac{\partial \Psi_{1}}{\partial t}+\nu_{1} \Psi_{1}\right)+\frac{c^{2}}{2 \omega_{1}} \frac{\partial^{2} \Psi_{1}}{\partial x^{2}}-\frac{\omega_{p}^{2}-\omega_{1}^{2}}{2 \omega_{1}} \Psi_{1} \\
& =\frac{\omega_{p}^{2}}{2 \omega_{1}} N \Psi_{1}-\frac{e}{4 \omega_{1} m_{e}} \Psi_{0} \frac{\partial E^{*}}{\partial x},
\end{aligned}
$$

where $v_{c}=\left(T_{e} / m_{e}\right)^{1 / 2}$ and $c_{s}=\left(Z T_{e} / m_{i}\right)^{1 / 2}$. Equations (37) - (40), provide improved approximation to the description of scattering instabilities as compared to Eqs. (15) - (17). They include several nonlinear terms and describe variations of the fields not in terms of slowly varying amplitudes $a_{0}, a_{1}, a_{2}$ but in terms of normalized ion density $N\left(x_{2} t\right)$, time enveloped electrostatic field amplitude $E$ and electron quiver velocities $\Psi_{0}$, $\Psi_{1}$ in the electric fields of electromagnetic pump and SRS backscattered wave, respectively. The complete electrostatic field reads $E=(1 / 2)\left(E(x, t) \exp \left(-i \omega_{p} t\right)+\right.$ c.c. $)$, and the electron quiver velocity in the electric fields of electromagnetic waves has the following form $V_{e}=(1 / 2) \sum_{r=0,1}\left(\Psi_{r} \exp \left(-i \omega_{r} t\right)+\right.$ c.c. $)$, where $\omega_{1}=-\omega_{p}+\omega_{0}$. By enveloping $E, \Psi_{0}, \Psi_{1}$ with respect to high frequency variations we keep in Eqs. (37)-(40) terms varying on the scale of ion motion. There is, however no restriction on the spatial variations of the physical quantities. As before $\nu_{0}, \nu_{1}$ describe damping coefficients of the pump and scattered light, respectively, and $\nu_{L}, \nu_{A}$ stand for linear damping operators of Langmuir fields and density disturbances, respectively. The latter contain Landau damping and effect of electron-ion collisions for electrostatic fields. The source terms $S_{L}, S_{A}$ are added in order to sustain the level of fluctuations of SRS active modes consistent with the thermal equilibrium values (cf. section Statistical Properties of the Source Terms $S_{\alpha}$ ) in the absence of the pump. The Zakharov equations are part of Eqs. (37)-(38). They are driven by the ponderomotive forces proportional to $\Psi_{0} \Psi_{1}^{*}(37)$ and $\left|\Psi_{0}\right|^{2}(38)$. The first one, which occurs on the right hand side of Eq. (37) drives SRS, and the second one is responsible for SBS interaction. The validity conditions for Zakharov equations, which are always satisfied in our simulations, are given by $|E|^{2} / 4 \pi n_{0}^{e} T_{e}<1$ and $|N|<1$. We have analyzed the wide range of plasma parameters with the common characteristic that the length of the system $L$ is always much longer than the damping length of Langmuir waves $V_{L} / \nu_{L}$. It is one of the important properties of short wavelength laser produced 

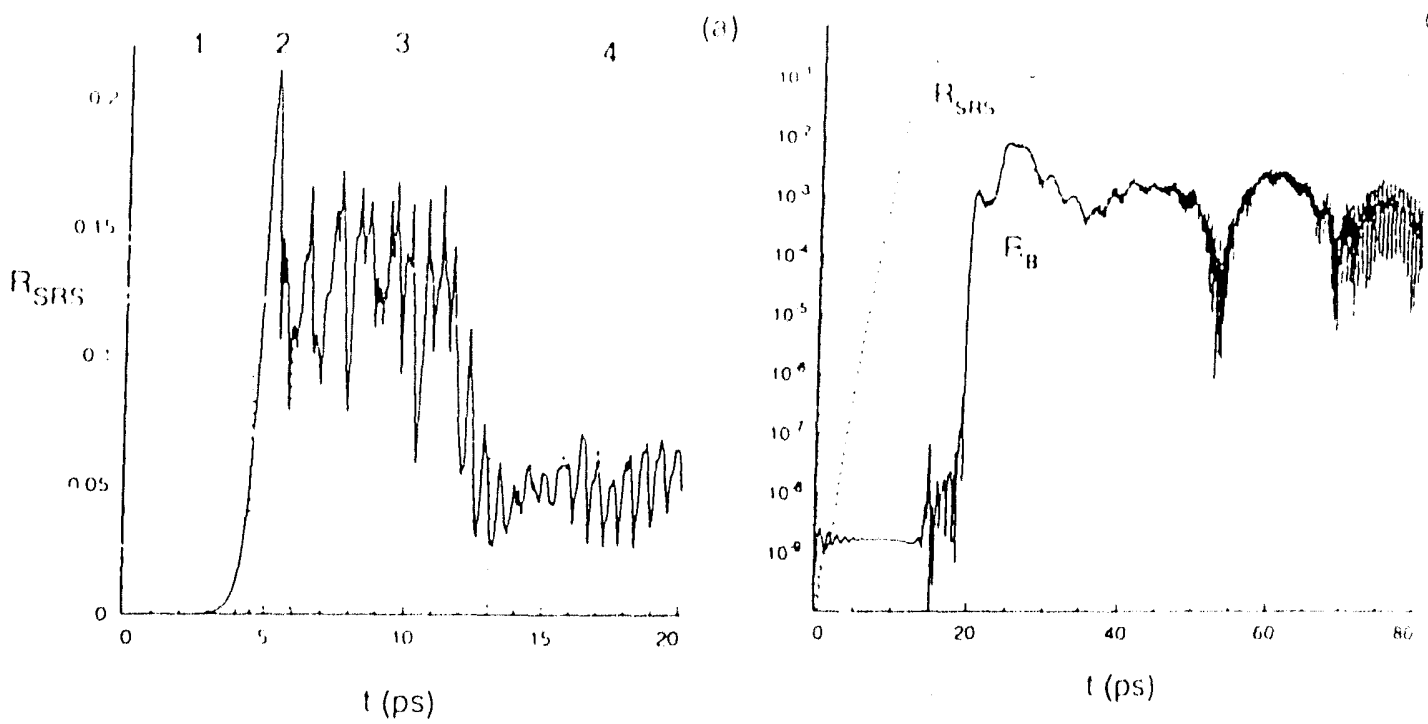

Figure 1: (a) Typical time history of the SRS reflectivity. Different lypes of behavion correspond to: (1) linear growth, (2) first saturation due to the Pl)l, (3) transient nonlinear regime characterized by the development of the PDI cascade and its disruption, (4) final saturation. (b) SRSS reflectivity and enhanced Brillouin reflectivity $R_{B}$ from the numerical solutions to Eqs. (37)-(40)for the following parameters: $n_{0} / n_{c}=0.2, T_{e}=1.5 \mathrm{kcV}, Z T_{c} / T_{1}=8, I=10^{14} \mathrm{~W} / \mathrm{cm}^{2}, L=60 \mu \mathrm{m}$

plasmas, where collisional damping on Langmuir waves plays an important, role

\section{SRS Reflectivity}

It has been shown in Ref. (12) that the main features of the one dimensional Zakharov - electromagnetic wave equations model (Eqs. (37) - (40)) can be divided into transient processes and the final saturation regime, which is independent of the initial conditions (cf. Fig. 1(a)).

During the transient evolution SRS grows at first linearly (phase (1) in Fig. 1) and saturates due to the onset of parametric decay instability of resonantly driven by SRS Langmuir wave (phase (2) in Fig. 1(a)). SRS never reaches the linear asymptotic regimes related to absolute or convective instabilities because the nonlinear effects due to PDI occur earlier. The linear growth rate is given by the gain factor (36) from the transient regime of the instability. The first nonlinear saturation corresponds to a strong flash of backscattered radiation (phase (2) in Fig. 1(a)). Next in the transient nonlinear regime ((3) in Fig. 1(a)) we observe development of the PDI cascade and its disruption due to different spatio-temporal evolution of SRS and PDI. In the asymptotic regime ((4) in Fig. 1(a)) SRS reflectivity saturates at the level which is well approximated by the scaling formula

$$
R=\frac{8}{27} \gamma_{0 R}^{2} L^{3} \nu_{A}^{k_{A}} \frac{\left(k_{L} v_{e}\right)^{2} \omega_{p}}{\omega_{0}^{2} \omega_{A} V_{0} V_{1}^{2}}
$$

In Eq. (41) $L$ is the plasma length, $\nu_{A}^{k_{A}}$ represents the Landau damping coefficient of the ion acoustic wave $\left(\omega_{A}, k_{A}\right)$ produced during PDI of the SRS driven Langmuir wave 


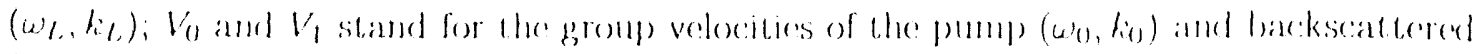
$\left(\omega_{1}, k_{1}\right)$ waves, respectively the homogenous shes growth rate condlicient is given by

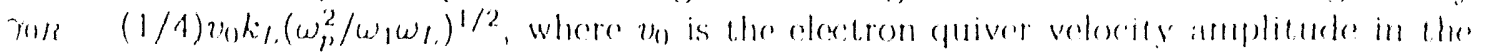
elecerie fiede of the pump wave. The waves participating in ShS inderation satisfy the

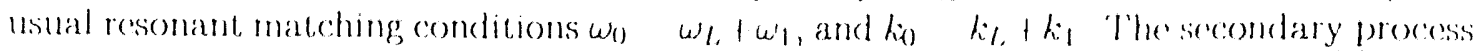
of the PI) corresponds to the resonant conditions: $\omega_{l} \omega_{l}^{(1)} \mid \omega_{A}$ and $k_{l} \quad k_{l}^{(1)}+k_{A}$, where $\left(\omega_{i}^{(1)}, k_{l}^{(1)}\right)$ eharacterizes the first component of the hangmuir wave cascade driven by PIJI. The matching conditions for PIDJ lead to the following solutions for the wave numbers involved in the first stage of the cascade: $k_{l}^{(1)}-k_{l}, \Delta k_{i}, k_{A}^{(1)}-2 k_{l}, \Delta k_{\text {, }}$ where $\left.\Delta k \quad(2 / 3) k_{l}\right) \sqrt{Z m_{r} / m_{\mathrm{t}}}$

The scaling expression for the reftextivity (41) has been derived without pump do pletion effects. Therefore it is only valid for small values of $R$. It also gives resonable predictions for the plasma density $n_{0}^{c} / n_{c} \leq 0.23$. Note that expression (11) does not depend on the damping coefficient, $\nu_{L}^{k_{L}}$ of Langmuir waves. The particular mechanism of Langmuir wave dissipation, i.e. for example Landau and collisional damping, or increased dissipation due to collapse processes (cf. Ref. (13|), does not directly affect SRS reflectivity but controls the spectrum of plasma waves and hot electron production. Fquation (41) can be rewritten in more explicit form

$$
R=6.610^{-7} I_{14} \lambda^{2}\left(\frac{L}{\lambda}\right)^{3} T_{e}^{2}\left(\frac{\nu_{A}^{k_{A}}}{\omega_{A}}\right) \Xi\left(n_{0}^{C} / n_{C}\right)
$$

where electron temperature $T_{e}$ is expressed in $\mathrm{keV}$, and $\Xi$ is a smoothly varying function of the electron density $n_{0}^{e} / n_{c}$,

$$
\Xi=\frac{\left(n_{0}^{e} / n_{c}\right)\left|1-\left(n_{0}^{e} / n_{c}\right)^{1 / 2}\right|\left|\sqrt{1-n_{0}^{e} / n_{c}}+\sqrt{1-2\left(n_{0}^{e} / n_{c}\right)^{1 / 2}}\right|^{4}}{\sqrt{1-n_{0}^{e} / n_{c}}\left|1-2\left(n_{0}^{c} / n_{c}\right)^{1 / 2}\right|}
$$

The expressions (41). (42) for the SRS reflectivity have been derived from scaling arguments [12], which includes an estimate of the Langmuir wave amplitude $E_{L}$ participating in the SRS interaction,

$$
E_{L}=E_{t h} \sqrt{\frac{2}{3} A}
$$

where $A=\gamma_{0 R}^{2} L / \nu_{L}^{k_{L}}\left|V_{1}\right|$ is the SRS amplification coefficient and $E_{t h}$ is the threshold of PDI

$$
\frac{E_{t h}}{\sqrt{4 \pi n_{0}^{e} T_{e}}}=\frac{16 \nu_{L}^{k_{L}} \nu_{A}^{k_{A}}}{\omega_{p} \omega_{A}}
$$

From expressions (44), (45) we can derive validity condition for the Zakharov equations in terms of relevant for SRS parameters

$$
1.2210^{-3} I_{14} \lambda^{2}\left(\frac{\nu_{A}^{k_{A}}}{\omega_{A}}\right)\left(\frac{L}{\lambda}\right) \Xi_{E}\left(n_{0}^{e} / n_{C}\right)<1,
$$

where $\Xi_{E}$ is the function of electron density,

$$
\Xi_{E}=\frac{\left|\sqrt{1-n_{0}^{e} / n_{c}}+\sqrt{1-2\left(n_{0}^{e} / n_{c}\right)^{1 / 2}}\right|^{2}}{\sqrt{\left(1-2\left(n_{0}^{e} / n_{c}\right)^{1 / 2} \mid\right.}}
$$

The limit (46) has been derived from the estimate of the electrostatic field amplitudes in the asymptotic saturation regime, where the fluctuations have already reached lower 


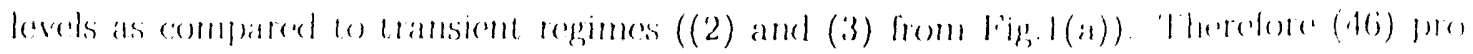
vides only meessiary condition for the validity of our muncrical calculations and it does

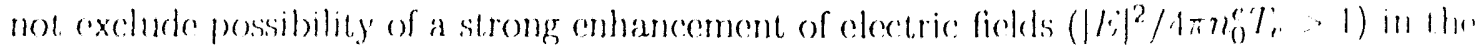

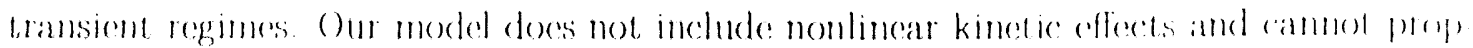

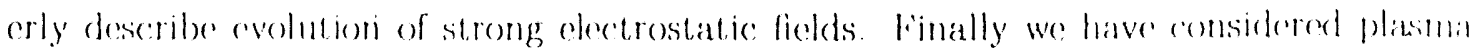
parameters, where SBSS cannot compete with SRR, i.e. approximately the gain of SBSt $G_{B}=\gamma_{0 / 3}^{2} L / V_{0} \nu_{A}^{2 k_{0}}$ is less than unity. In fact this limitation is stronger than neesssary, in particular for short pulse interaction experiments when the long time behavior of SBSS is irrelevant.

\section{Laser Light Scattering from SRS Produced Fluctuations}

The nonlinear evolution of langmuir waves, which is responsible for the saturation of SRS, results in a broad spectra of the electrostatic fluctuations. The PDI cascade, tocalization of Langmuir waves and spatial evolution of the instabilities produce enhancerd fluctuation levels which in turn can scatter the electromagnetic pump via Brillonin, forward Raman, and/or blue-shifted Raman interactions. These processes can be particularly important for the short pulse laser plasma interaction experiments, where fast growing SRS and subsequent PDI of Langmuir waves can enhance ion fluctuations by several orders of magnitude on the time scale when, for example, ordinary SBS has no chance to grow We will concentrate in this review on the description of the enhancement of the Brillouin scattering by the backward SRS (cf. Ref. |14|), which can produce the most pronounced and unique effects in experiments.

Figure 1(b) illustrates the time evolution of SRS reflectivity' together with the enhanced Brillouin reflectivity $R_{B}$. For the conditions of this run $\left(n_{0} / n_{c}=0.2, T_{e}=1.5 \mathrm{keV}\right.$, $Z T_{c} / T_{i}=8, I=10^{14} \mathrm{~W} / \mathrm{cm}^{2}, L=60 \mu \mathrm{m}, \lambda=0.5 \mu \mathrm{m}$ ) we do not observe a temporal growth of the ordinary stimulated Brillouin scattering. The sudden onset of the backscattered Brillouin signal lakes place during the intermediate nonlinear evolution of SRS (phase (3) from Fig. 1(a)) and it is related to the broad ion acoustic spectrum of nuctuations produced at that time. It coincides with the development of high components of the PDI cascade and with the formation of continuum in the fluctuation spectra. The level of Brillouin scattering depends on the enhancement of the ion acoustic spectrum. In particular on the level of fluctuations produced at the wave number $k=k_{A}^{B}=2 k_{0}$, which partlcipates in the Brillouin interaction. The extend of the enhanced region in the ion wave spectrum

scales as the overall number of steps in the PDI cascade. It is proportional to the ratio $E_{L}^{2} / E_{t h}^{2}$, i.e. for the saturated SRS the number of steps in the PDI cascade is simply $\frac{2}{3} A$ (cf. Eq. 44). Using this estimate, we may derive simple strong enhancement criteria for Brillouin scattering, in terms of the backward SRS amplification coeficient

$$
\frac{2}{3} A>\left|k_{A}^{B}-k_{A}\right| / 2 \Delta k
$$

where $2 \Delta k$ is the separation between ion modes produced by PDI. Figure 2(a) shows enhanced spectra corresponding to the late moment in time of the run illustrated in Fig. 1(b). The enhanced part of the spectrum is dominated by broad, but distinct peaks corresponding to modes produced by PDI. The value of $k=k_{A}^{B}=0.22 k_{D}$ is localed very close to the fourth component of the PDI cascade which is a mode propagating in the opposite direction to the pump. Electromagnetic radiation which is scattered due to interaction with this mode would produce primarily the blue shifted component observed in the frequency spectrum (cf. Fig. 2(b)). The contour plot (Fig. 2(b)) displays the time evolution of the spectral intensity of Brillouin backscattered radiation normalized to the pump intensity at $\omega=\omega_{0}$. The shift of red and blue maxima corresponds approximately to the ion acoustic 


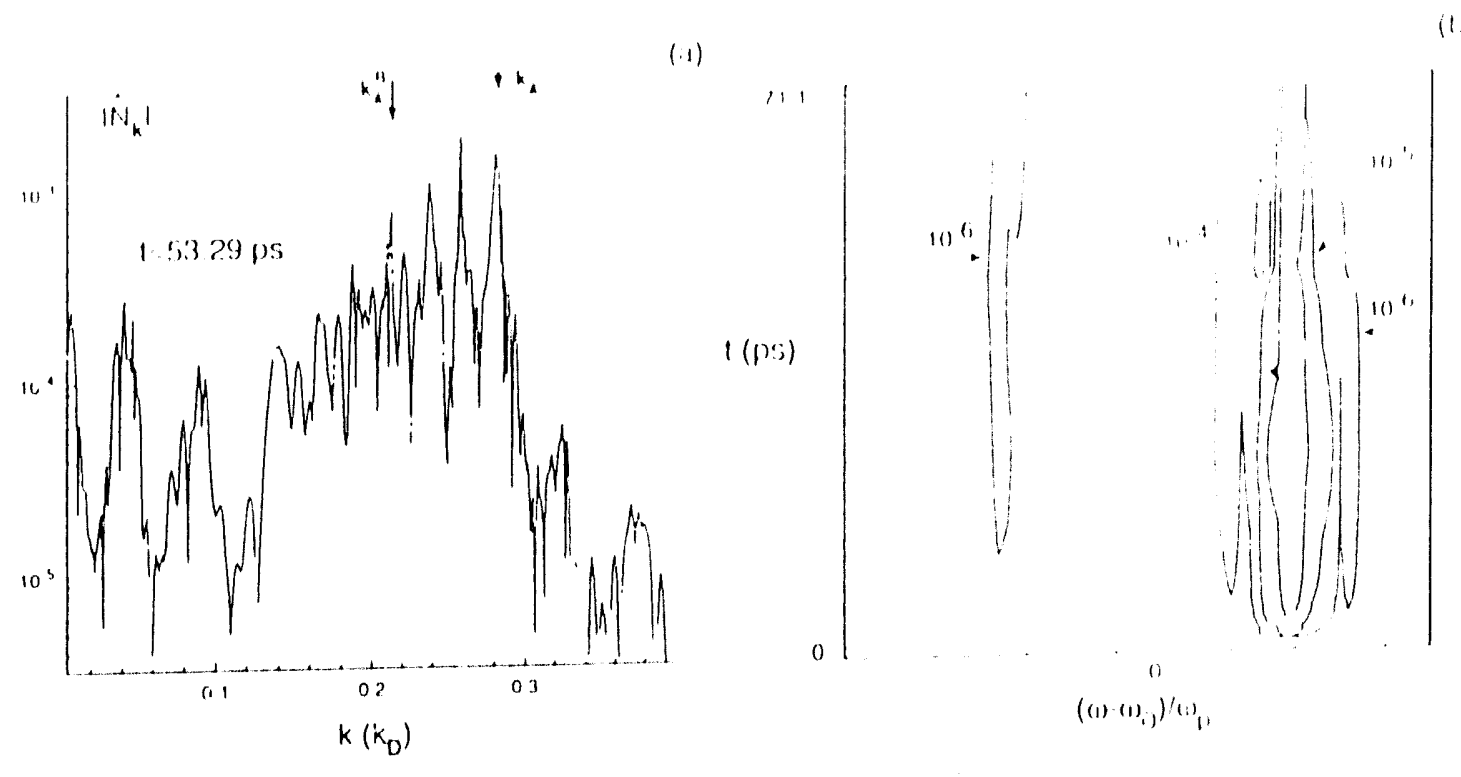

Figure 2: Numerical simulations for the parameters of fing (b). (a) normalized

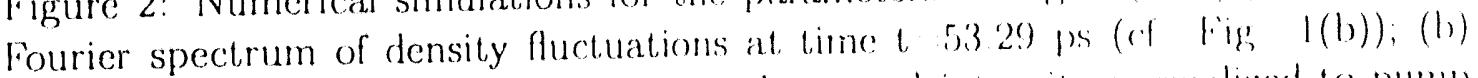
contour plot of the Brillouin backscattered spectral intensity nomalized to pump) intensity.

frequency $2 k_{0} c_{s}$ : Since the enhanced part of ion wave spectrum is domunated by almost discrete peaks small change in plasma parameters can move $k_{A}^{\prime \prime}$ component closer to the mode propagating along the pump and produce strongly enhanced red shifted spectrum. There is also a continuos part of the density spectra, which will enhance blue and reat shifted electromagnetic waves to the similar levels. The presence of blue and red shifted components in the spectra of the scattered light is a characteristic feature, which can help) to identify this process experimentally.

\section{SCATTERING INSTABILITIES INVOLVING ION WAVES}

In the previous Section we have discussed the stimulated Raman scattering which is a resonant instability involving electron plasma waves. We turn our attention now to parametric processes corresponding to the interaction between electromagnetic and ion waves They are: stimulated Brillouin scattering (SBS), filamentation (FI) and self focusing (SFI) instabilities $|1,2|$. The ion wave instabilities are of a major concern for the laser fusion schemes, because of their potential capability to reflect a substantial part of the incident laser energy, spoil the compression symmetry, and stimulate other undesirable nonlinear processes $\{15,16 \mid$. Experiments and theoretical analysis of these instabilities on the short time scale in well controlled plasmas $|5|$ are helping to understand physies of these processes, including their mutual interaction and nonlinear evolution. In this review we will emphasize the common origin of SBS, FI and SFI, related to the ion dynamical response to ponderomotive forces of an electromagnetic and/or a thermal origin. The differences correspond to the nature of ion response: SBS excites resonant ion acoustic waves, while FI and SFI produce non resonant ion density disturbances.

The existing theories of ion wave scattering instabilities fail to explain such commonly 


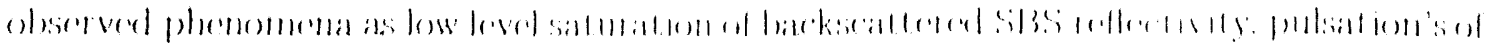

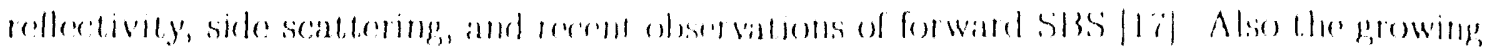

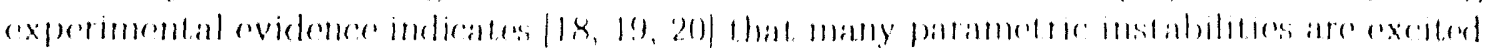

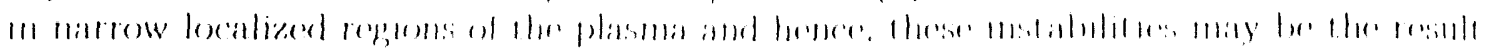

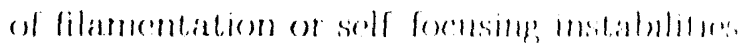

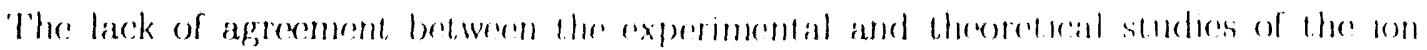

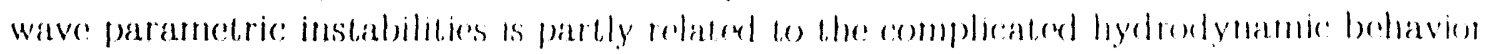

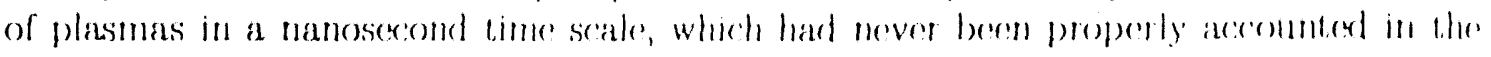

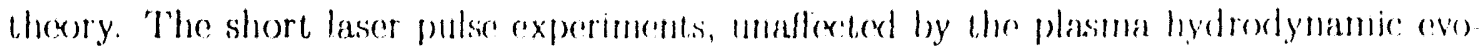

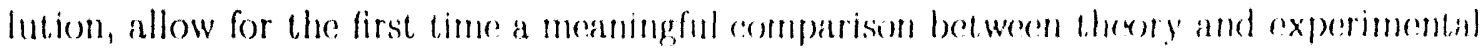
observations. The other origin of the discrepancy are the complieated spatial profiles of

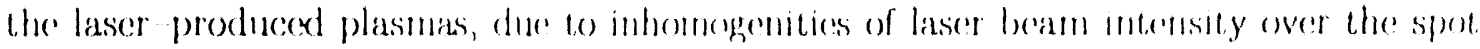
size. 'The new experimental terhngue of laser beam smoothing |2ll enables us to overcome this difficulty and to preform plasma with well controlled and rather platn profiles Therefore the combination of the laser beam smoothing with the subpicosecond plasmat diagnostic and short laser pulses opens quate new possibilities in thr understanding of ion wave parametric instabilities.

In this section we will describethe main characteristics of the ion mode parametric: instabilities at the linear stage and possible mochanisms of their momlimear saturation, to gether with the results of numerical simmlations.

\section{Basic Equations}

We consider an homogeneons plasma slab in eartesian geometry with tho jon density $n_{0}^{\prime}$ and electron density $n_{0}^{e}-Z n_{0}^{\prime}$, where $Z$ is the average ion charge. $\wedge$ beam of $s$ polarized electromagnetic wave of frequency $\omega_{0}$ is incident on the plasma slab at $x$..: 0 and propagates along $x$-direction. The time enveloped of the clestric ficld $\mathrm{E}$ of the transverse electromagnetic wave polarized along $z$-axis, and the ion density perturbation $\delta N=n_{i} / n_{0}^{i}-1$, satisfy the system of efuations derived from the standard set of the Maxwell and plasma hydrodynamic equations. Neglesting the dispersion and nonlinear effects on ion acoustic wave propagation, one obtains $(22,23)$ :

$$
\begin{gathered}
\left(2 i \frac{\omega_{0}}{c^{2}} \frac{\partial}{\partial t}++\nabla^{2}+k_{0}^{2}+i \frac{\nu_{e i} \omega_{0}}{c^{2}} \frac{n_{0}^{e}}{n_{c}}\right) E=\frac{\omega_{0}^{2}}{c^{2}} \frac{n_{0}^{c}}{n_{c}} E \delta N, \\
\left(\frac{\partial^{2}}{\partial t^{2}}+2 \hat{\Gamma}_{s} \frac{\partial}{\partial t}-c_{s}^{2} \nabla^{2}\right) \delta N=\frac{Z}{16 \pi m_{s} n_{c}} \nabla^{2}|E|^{2},
\end{gathered}
$$

where $k_{0}=\left(\omega_{0} / c\right) \sqrt{1-n_{0}^{e} / n_{c}}$ is the wavenumber of the laser pump, the operator $\hat{\Gamma}_{s}$ models the damping of ion density fluctuations, $c_{s}=\sqrt{\left(Z T_{c}+3 T_{i}\right) / m_{i}}$ is the ion sound speed, $c$ is the speed of light, $n_{c}=m_{e} \omega_{0}^{2} / 4 \pi e^{2}$ is the critical density, and $\nu_{e i}$ is the electron-ion collision frequency describing the absorption of the electromagnetic waves.

The two equations (49), (50) describe the coupling between high frequency electromagnetic waves and low frequency plasma density via the ponderomotive force tern on the right hand side of Eq.(50). It is known, that in addition to ponderomotive effects the ion waves can be excited by the inhomogeneous plasma heating. Thermal effects can be important for SBS and filamentation instabilities especially in the regime of parameters where the electron mean free path is comparable with the characteristic wavelength of ton fluctuations. The importance of these processes has been recognized recently as a result of the better understanding of nonlocal thermal transport effects $|24|,|25|$. However in this review we will deal only with the ponderomotive case and leave for the future the discussion of the thernal instabilities, which we are currently studying within the context 


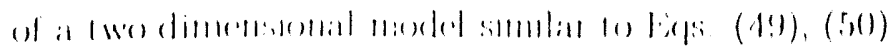

Linear 'Theory of Lon-Wane Scattering Instahilities

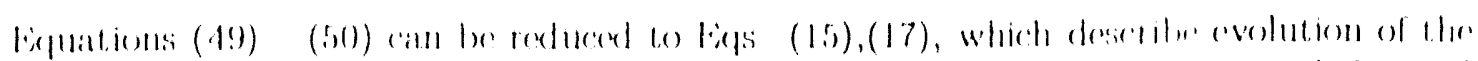

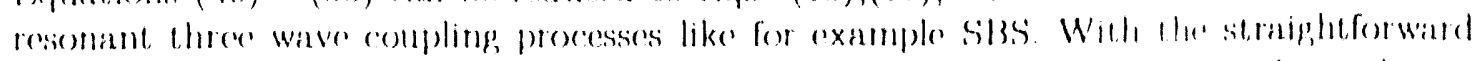

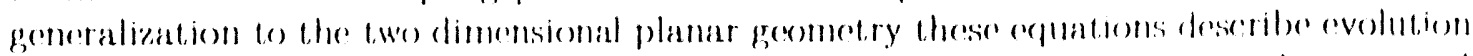
of slowly varying amplitudes of the waves participating in backward or side seatlerey sibs. The linear analysis of these interactions lead to the results summarizod in a pre vious section. The scattering in the forward direction grows stower than the attering producing electromagnetic: radiation propagating in the backward direstion. However because the forward sks' involves ion waves of the relatively smaller wave mumbers they are weakly damperd and the seattering can reach higher level in the asymptotic linear regime characterized by stationary amplification coeflicient (27).

Our model (19), (50) describes sound waves in the linear approximation, but accouts for the competition between the backward and side scattered SRS, as woll at the filamentation instability. In order to understand the coexistence of these instabilities, one must. describe not only the linear growth but also the initial levels of fluctuations. The results of our 21) model show that the thennal levels of sound waves underestimate the real noise amplitudes, in particular for the stationary fluctuations with the transverse wave vectors to the direction of laser pump propagation. These fluctuations, which are increased by the incident laser beam participate in the filamentation instability. They can enhance this relatively slowly growing interaction to the level were it competes with sibs, even on the scale of short pulse laser experiments.

The expression for the filamentation growth rate follows from liss (49), (50) if we assume that the scattered waves propagating obliquely to the pump have no frequency shift $\left(k_{\perp} \ll k_{0}\right)$ :

$$
\left.E(\mathrm{r}, t)=\left|E_{0}+E_{-} \exp \left(-i \mathrm{k}_{\perp} \mathbf{r}_{\perp}\right)+E_{+} \exp \left(i \mathrm{k}_{\perp} \mathrm{r}_{\perp}\right)\right| \exp \left(i k_{i}\right) l\right)
$$

The filamentation instability, similarly to SBS, exhibits complicated spatial--temporal behavior before the convective saturation. In the transient stage it is diflicult to separate the filamentation from the forward SBS, because their characteristic growth rates are comparable with the Brillouin frequency shift and therefore, both of them lead to the non stationary disturbances of the pump beam. However, the stationary amplification coefficient for FI differs considerably from that of SBS (27). According to Ref. $[15,27 \mid$ in the case of the thermal filamentation and classical heat transport $\left(k_{\perp} \lambda_{e}<1\right.$, where $\lambda_{e}$ is the electron mean free path) the amplification takes place for $k_{\perp}<k_{c r}^{(t h)}$ where $K_{c r}^{(t h)}$ $\sqrt{k_{0} / \lambda_{e}} \sqrt[4]{n_{0}^{e}\left|E_{0}\right|^{2} / 4 \pi n_{c}^{2} T_{e}}$. In that case the coefficient of the exponential amplification in the plasma slab of the length $L$ has the following form :

$$
G_{t h} \approx\left(K_{c r}^{(t h)}\right)^{2} L / h_{0} .
$$

The ponderomotive FI takes place for the small-scale inhomogeneities $\left(k_{1} \ll \lambda_{e}\right)$ when $k_{\perp} \approx K_{c r}^{(p)} \approx k_{0} \sqrt{n_{0}^{e}\left|E_{0}\right|^{2} / 4 \pi n_{c}^{2} T_{e}}$. The corresponding amplification coefficient is $G_{p} \approx$ $\left(K_{c r}^{(p)}\right)^{2} L / k_{0}$.

All these expression for the filamentation instability are relevant if the laser beam width $a$ is much larger than the characteristic filamentation scale, $k_{\perp} a \gg 1$, and FI results in splitting of the whole beam into several nearly independent filaments. If the beam size is smaller than the filamentation scale than the whole beam self focusing (SFI) can take place. The condition of the thermal SFI onset is $G_{t h}>1$ for $a \gg \lambda_{e}$. The ponderomotive SFl is in effect for narrower beams $a \ll \lambda_{e}$ and starts 10 work if $K_{r}^{(p)} l>k_{0} a$. In the 


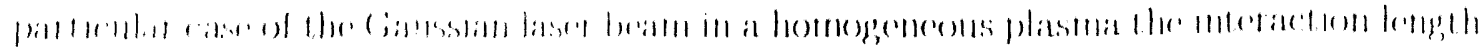

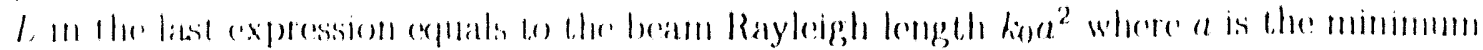

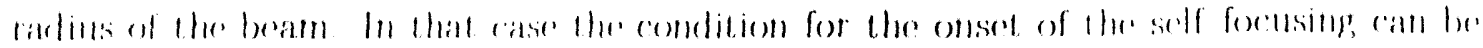
w11 1011 is: $1, f \cdot 1$ wher,

$$
\text { (1, }\left(k_{10} n\right)^{2}\left(n_{n}\left|H_{0}\right|^{2} / 8 n n_{c}^{2} T_{c}\right)
$$

1. Hor solf fochsing parametor $|27|$

'Two dimonsional Simulations of Ion-Wave Parametric Instabilities

In most theoretical studies the different ion wave scattering instabilitics have been investigated separately, while in experiments, they can occur simultanconsly. 'lherefore. in this section the competition between SBS in different directions, filamentation, and self focusing instabllitios will be discussed for the case of a ponderomotive conpling. At first we will describe forward and backward SBS competition and next, we will discuss effects of the laser beam self focusing on SBS.

The system of equations (49), (50) has been solved in two dimensional cartesian geometry with non periodic outgoing boundary conditions. The incident Gaussian like laser beam enters at the boundary $x$. 0 and propagates along the $x$ axis. The length of interaction region $L$ is assumed to be less or comparable to the Rayleigh length of the incident laser beam $k_{0} a^{2}$. This choice corresponds approximately to the hot spots in the laser beams in an underdense plasma corona of ICF targets.

For the adequate comparison with experiments the results of simulations have been represented in terms of the emission field outside the interaction volume. According to the standard Halmholtz--Kirchhoff representation of the radiated field we have calculated the electric field $E(\rho, t)$ in a far field (Fraunhofer) zone at a distance $\rho \gg k_{0} a^{2}$ from the interaction region. The emitted power in a unit angle has been represented as $Q(\theta, t)$ $c \rho|E(\rho, t)|^{2} / 8 \pi$, where $\theta$ is the emission angle with respect to the propagation axis of the incident beam. Along with the angular distribution $Q$, the integral quantity of the scattered light - the reflectivity in backward semicircle - have also been calculated:

$$
R_{b}(t)=P_{0}^{-1} \int_{\pi / 2}^{3 \pi / 2} d \theta Q(0, t)
$$

where $P_{0}$ is the incident beam power. $R_{b}$ is a convenient characteristics of SBS, describing its most detrimental feature for ICF applications, and allowing for interesting comparisons with one dimensional calculations.

\section{Forward-backward SBS interaction.}

The numerical solutions $|26|$ to Eqs. (49),(50) have shown strong dependence of the angular distribution of SBS emission on the ion acoustic mode damping and the geometry of the interaction region. The characteristic temporal dependence of the backscattered reflectivity $R_{b}$ is shown in Fig. 3(a) for the case of a relatively strong damped ion acoustic waves $\Gamma \equiv \Gamma_{s}\left(2 k_{0}\right) / 2 k_{0} c_{s}=0.06$, and in $F i g$. $3(\mathrm{~b})$ for the case of weak damping $\Gamma=0.03$. As we will see later, the angular distribution of the scattered radiation can be very complicated and only in the case of a narrow pump beam the main signal is emitted in the backward direction. The dotted curves in Figs. 3 represent results obtained from the one dimensional (1D) version ofthe code. For a comparison, the dashed curves displayed in Fig 3 have been obtained from ID runs exploiting driven Korteweg-de Vries (KdV) equation [28] for the ion density perturbations. The one-dimensional results correspond closely to the case of narrow pump $(a<L)$, whereas the wide beam runs $(a>L)$ exhibit much 

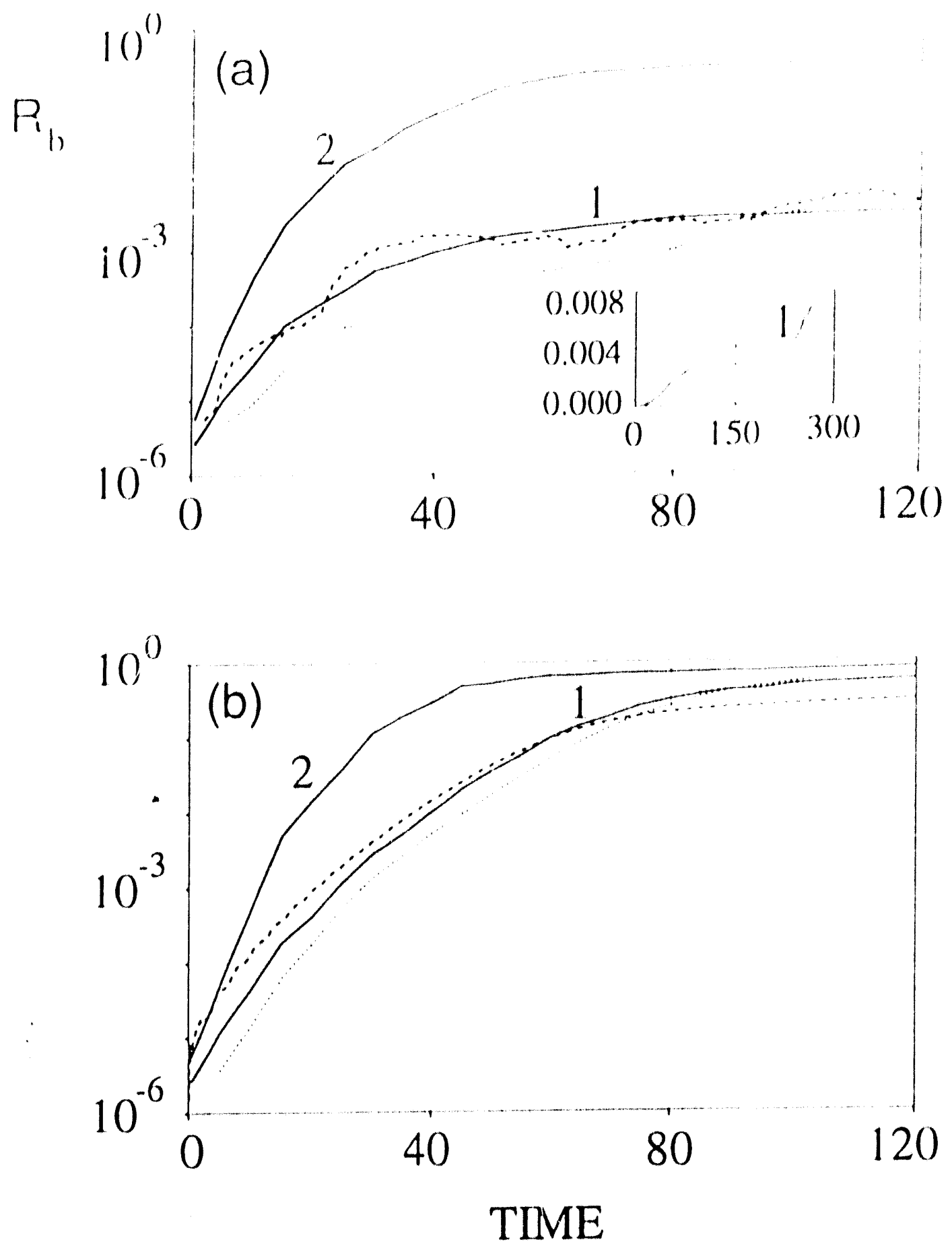

Figure 3: 'Temporal dependence of the reflectivity in rear semicircle $R_{b}\left(k_{0} c_{s} t\right)$ for the case of $(a)$ strong $(\Gamma=0.06)$ and $(b)$ weak $(\Gamma=0.03)$ ion acoustic wave damping. The other parameters of the simulations are the following: $\left|E_{0}\right|^{2} / 8 \pi n_{e c} T_{e}=0.045$, $n_{i} / n_{1 c}=0.2$, interaction length $L=35.5 \lambda_{0} ; a=10 \lambda_{0}$ for the curves 1 , and $a=50 \lambda_{0}$ for the curves 2 . The dotted lines correspond to $1 \mathrm{D}$ results, and dashed curves has been obtained from ID KdV theory for the same parameters. The insert in panel (a) displays long-term behavior for curve 1 in lincar scalc. 

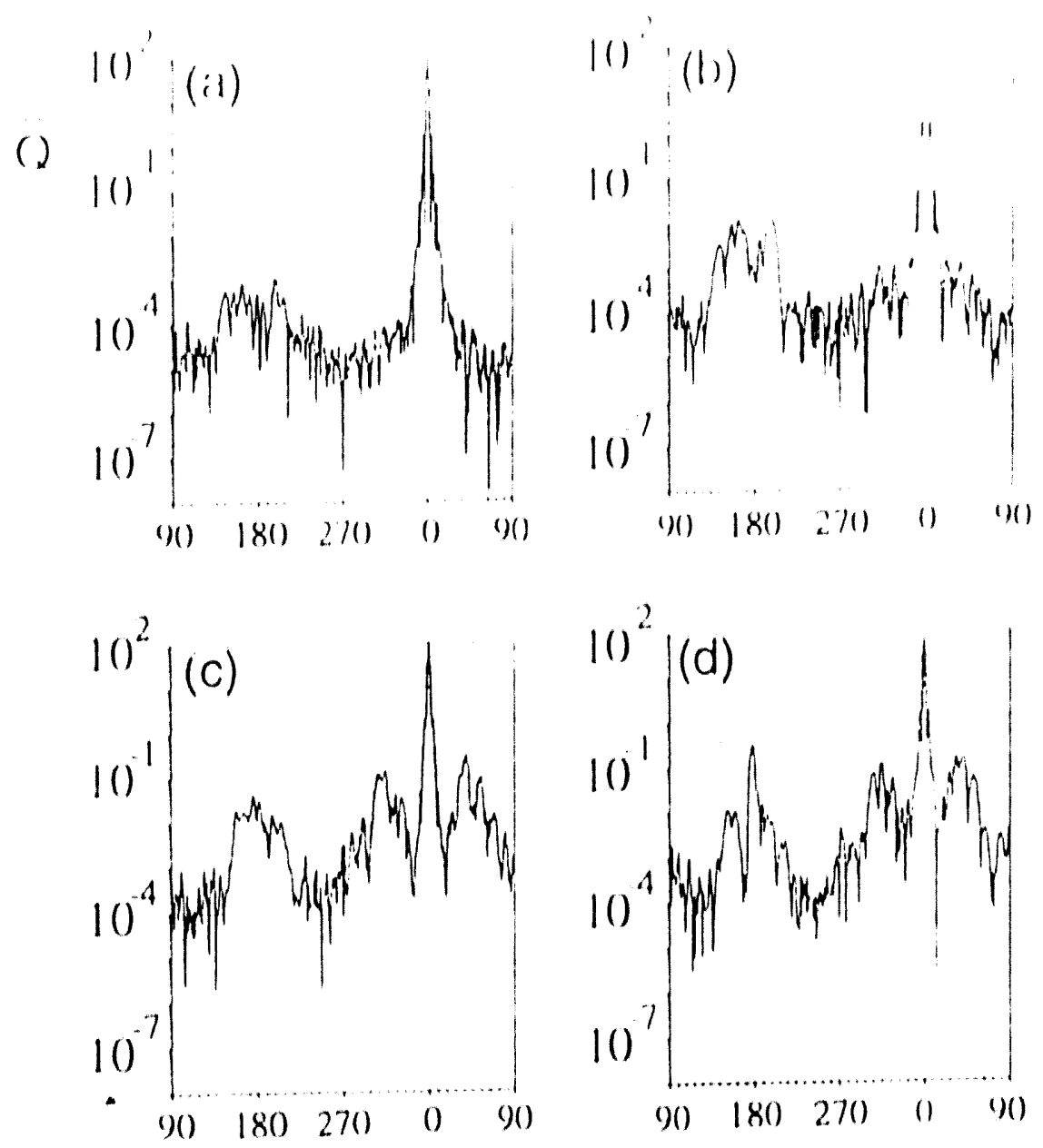

Scattering Angle (degrees)

Figure 4: Angular distribution of the electromagnetic wave power emitted from the plasma slab in the far field zone $\dot{Q}=Q(0, t) /\left(n_{1} m_{1} c_{s}^{2} c / k_{0}\right)$ for the parameters of curve 1 in the ligs. 3 (a) at lime $k_{0} c_{s} t=15(a), 45(b), 165$ (c), and 285 (d)

higher reflectivity. It is interesting to note that 21) simulations display more complicatial long time behavior: of. insert panel in Fig. 3 (a), where a backscatered sigmal starts 10 grow up again after a long period of the apparent saturation.

Additional information about physical processes lending to the time evolution of the reflectivity (Fig. 3) are shown in Figs. 4 and 5 . They show the angular distribution of the scattered electromagnetic radiation in the far field (liraunhofer) zone. The transmittur light is emilted in a narrow cone $\Delta \theta \approx \lambda_{0} / a$ which is less than $10^{\circ}$ for the cise of narrow pump (curves 1 in ligig. 3), and about $1^{\circ}$ for wider pump (curves 2 in Fig. 3) 'The rest of the electromagnetic radiation in the far field zone is produccel due to scattering by the ion density perturbations. 'The initial level of scattering by the random density fluctuations is about $Q_{0} \sim 10^{-5} \%$, and does not depend on the angle 0 . For the case of relatively long interaction regions $(a<b)$ (cf. Fig. $A(a)$, (b) and s) (a)), tho backward SIBS grows lirst and is emitted in a relatively large cone angle $\left.\delta O_{b} \approx t^{-1}\right)^{-1}(a / L)$. Note that the small scale $\left(\sim 1^{\circ}\right)$ modulation pattern in far field plots is the conseguence of finite grid size used in the calculations. The large scale $\left(\sim 10^{\circ}\right)$ modulations are the result of scattered light diffraction due to density channel formed by the incident pump beant A very important parameter, responsible for the evolution of senttering, is tho stationary amplification coeflicient $G_{b}$ for backward SBS (eq.(27)). If the ton acoustic damping. 


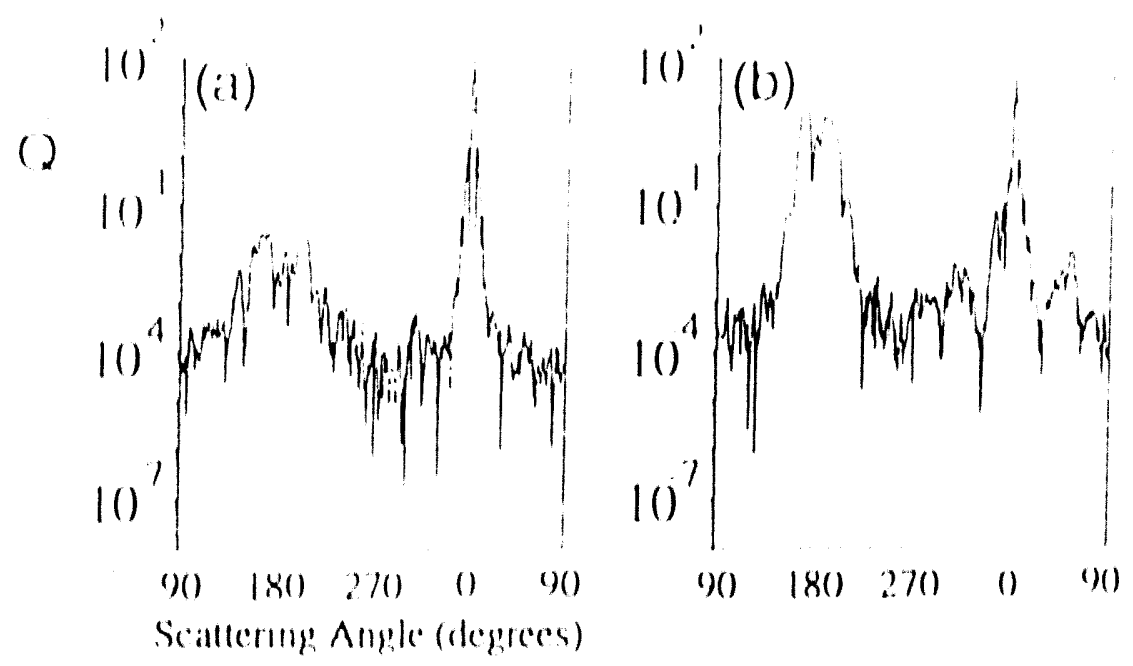

Figure is Angular distmbutem of electromagnetic wave power emonted from the

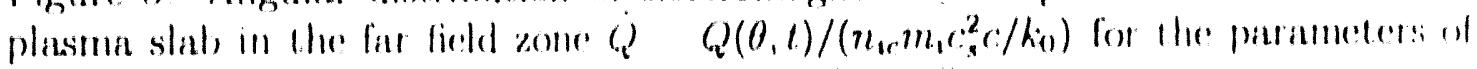
curve I in lis is (b) all time hines 30 (a) and 180 (b)

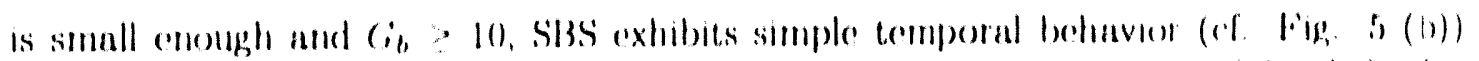
backscatterest light depletes the pumpl beam and saturates at a relat.uvely high level In that rase, two dimensional effects are not very important and the total backward roflectivity is closer (1) one dimentional calculations as can be seen from fing. 3 (b). The difference between 21) results (curve 1), or dotted curve corresponding to linear 11) simulations. and dashed curve in this figure may be atcributed to the fon hydrodynamic: nonlinearitios The latter inhibit the growth of the ion waves and are taken into necount in the $11 \mathrm{O} \mathrm{KdV}$ theory

After the backward SBSS saturation, side scattering grows to the levels about two orders of magnitude higher than intial ones, and eventually saturates. T'he cause of this saturation is demonstrated in Fig. 6 which shows the electric field and density distributums inside the plasmn. In Fig. $6(n)$, one can see that regions of back seattering and side scattering are separater, and due to the pump depletion, side scatcering cannot grow to the level that the linear theory predicts.

If the damping of ion acoustic mode is strong enough and backscattering saturates at a low level without noticeable pump depletion, then the scattering in other directions have a chance to grow. This is illustrated in Fig. 4 (c) where the forward scattering in angles $0 \sim 40^{\circ}-60^{\circ}$ reaches even larger levels than backscattering. The characteristic angles of forward entssion are defined by the Fourier spectrum of density disturbances produced by the pump beam during the process of the density channel formation.

The growth and saturation of forward SBS has important effect on backscattering One can see in Fig. 1 (d) that additional back reflection shown in insert panel of Fis? 3 (a) has cualitatively difforent angular shape: its angular divergence is approximately the same as the pump beam's one. The near field distribution can help to understand the cause of this collimated backscatter. The forward and backward scattering procosses are spatially separated because of convective nature of these instabilities and due to the propagation of scattered waves in opposite directions. The density disturbances relater to backscattering are localized near the entrance boundary (see Fig. 6 (b)). 'The density fluctuations produced by forward SBS reach their maximum near the forward boundary and can act as an additional seed for backscattering. Due to diffraction of the scathercel waves on these density inhomogenelties, the preference in amplification among all possible 

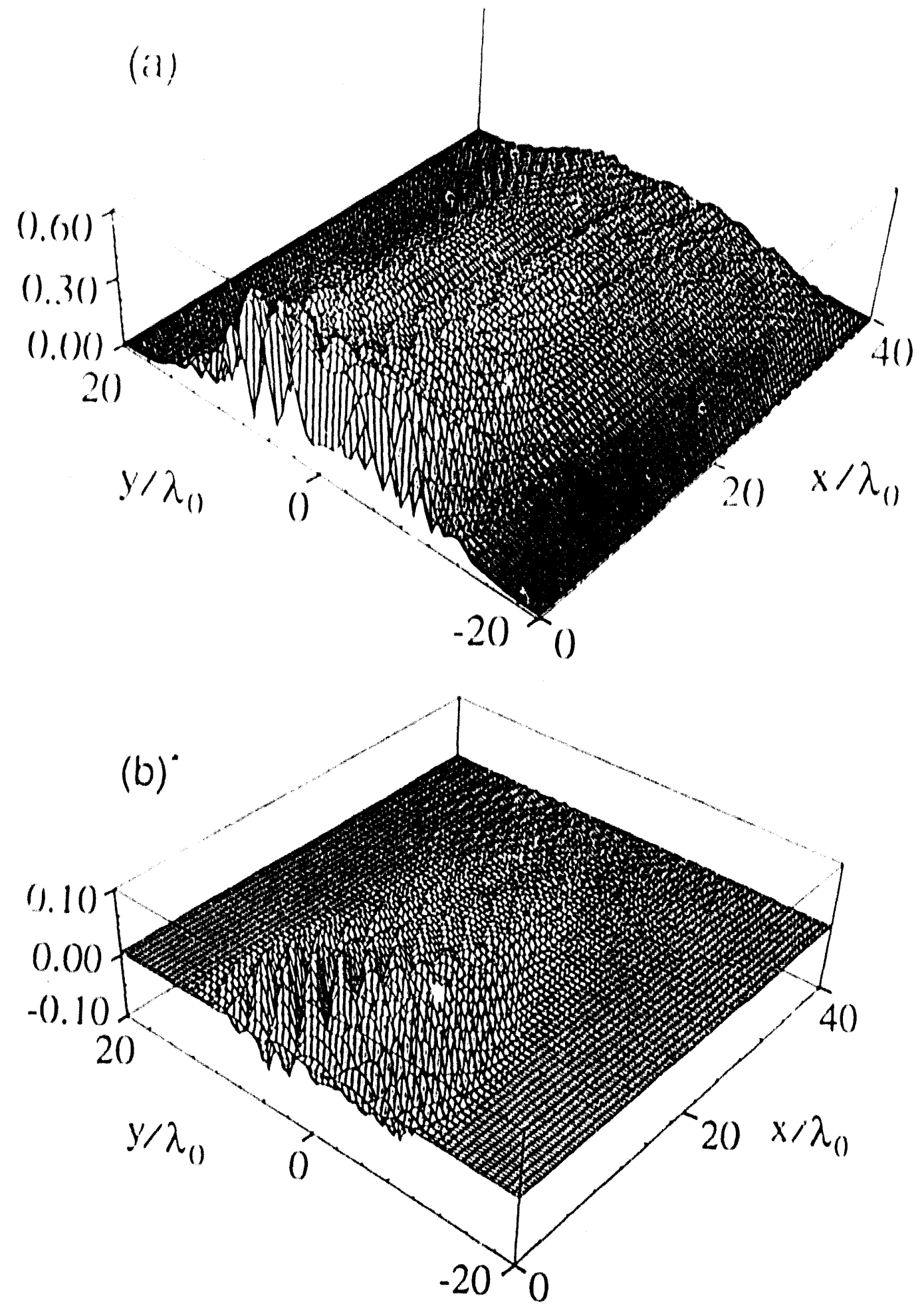

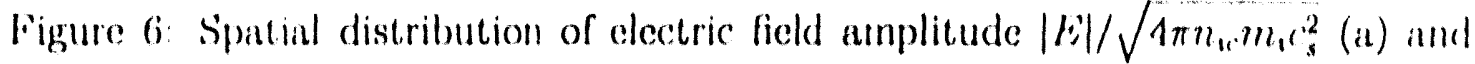
the ion density fluctuations $\delta N$ (b) in the plasma slab for the parameters of curve 1 in Fig. $3(b)$ at time $k_{0} c_{s} t=180$. 


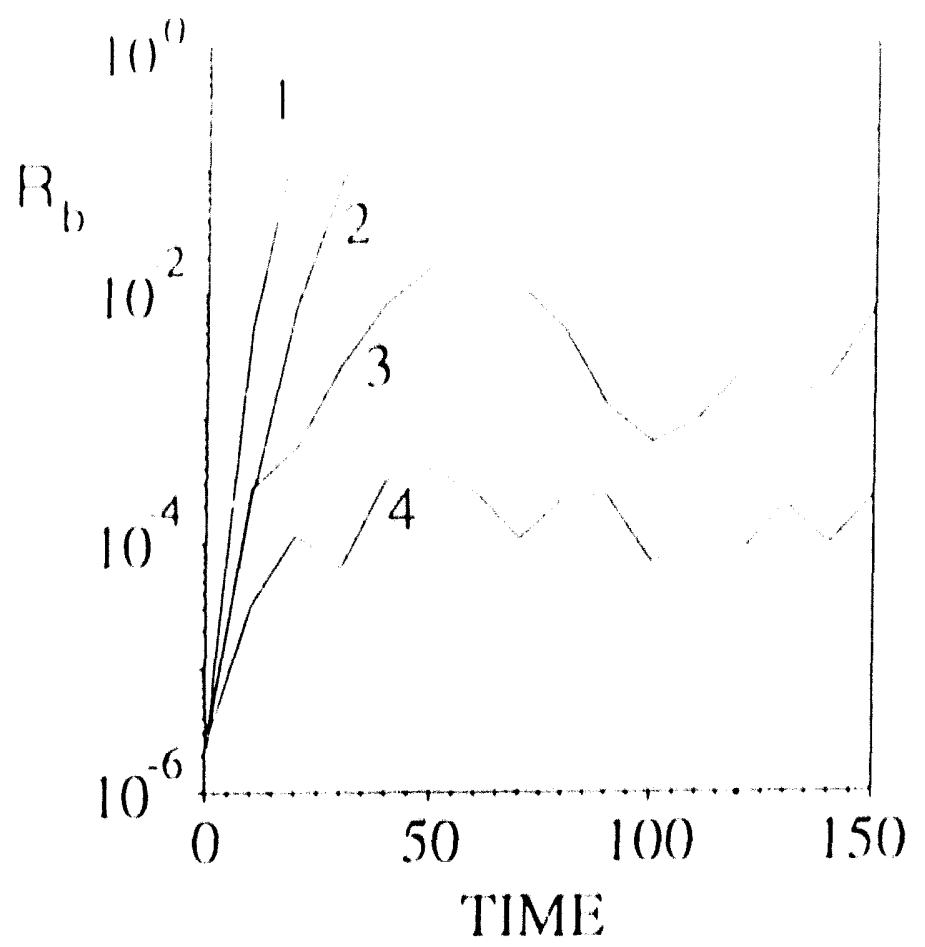

Figure 7 Temporal dependence of the reflectivity in the rear hemicirele $R_{b}\left(k_{1} c_{2}, t\right)$ for the different ion acoustic wave damping: I'- 0.03 (curve 1), 11 I (2), (0.2 (3), and 0.3 (1). The other parameters of the simulations are the following $\left|K_{0}\right|^{2} / 8 \pi n_{r e} T_{\text {e }}$. $0.045, n_{1} / n_{\mathrm{tc}} \mathrm{c}^{\circ} \quad 0.5, k_{0} \Omega=20$; interaction length $l=55.5 \lambda_{0}$ for curve 1 , and L. $45.5 \lambda_{0}$ for curves $2,3,4$.

structures has the backward propagating wave, whose shape is approxumately the same as the pump beam shape. This wave has smaller losses and therefore can gain more cnergy These arguments, which are similar to that used in the light phase conjugation theory $|29,30|$, elucidate the reasons for the collimated backscattering generation at the late stage of SBS temporal evolution. In other words, the forward SBS produces the condi. tions for the effective electromagnetic mode competition, which are absent in an initially homogeneous plasma. Therefore, these simulations demonstrate the possibility and provide the necessary conditions of the phase conjugation offect in laser plasma interaction

\section{SBS from intense localized beam structures.}

In this section we will discuss simulations when the ponderomotive self focusing instab)l. ity is allowed in the interaction region. Figure 7 demonstrates the temporal dependence of the backward reflectivity (53) for several runs with different ion acoustic damping. The self-focusing parameter (52) $\alpha_{s f}=9$ is the same for all curves. The damping parameter $\Gamma$ controls backward SBS amplification coefficient and doesn't affece self focusing. In the case of weak damping (ef. curves 1 and 2 in ligg. 7), backscattering apparently domnates and leads to a high level reflectivity, which is determined by the pump depletion Self-focusing plays the secondary function in this run. This is why curves 1 and 2 in Fig, 7 look simflar to the curves displayed in Fig. 3. Bscause of the fast growing backscat. tering, the intense electric fields are localized near the entrance line and the conventional self-focusing has not enough length for its excitation. However, the far field distributions exhlbit few angular sidebands in forward and backward directions which can be attributed 

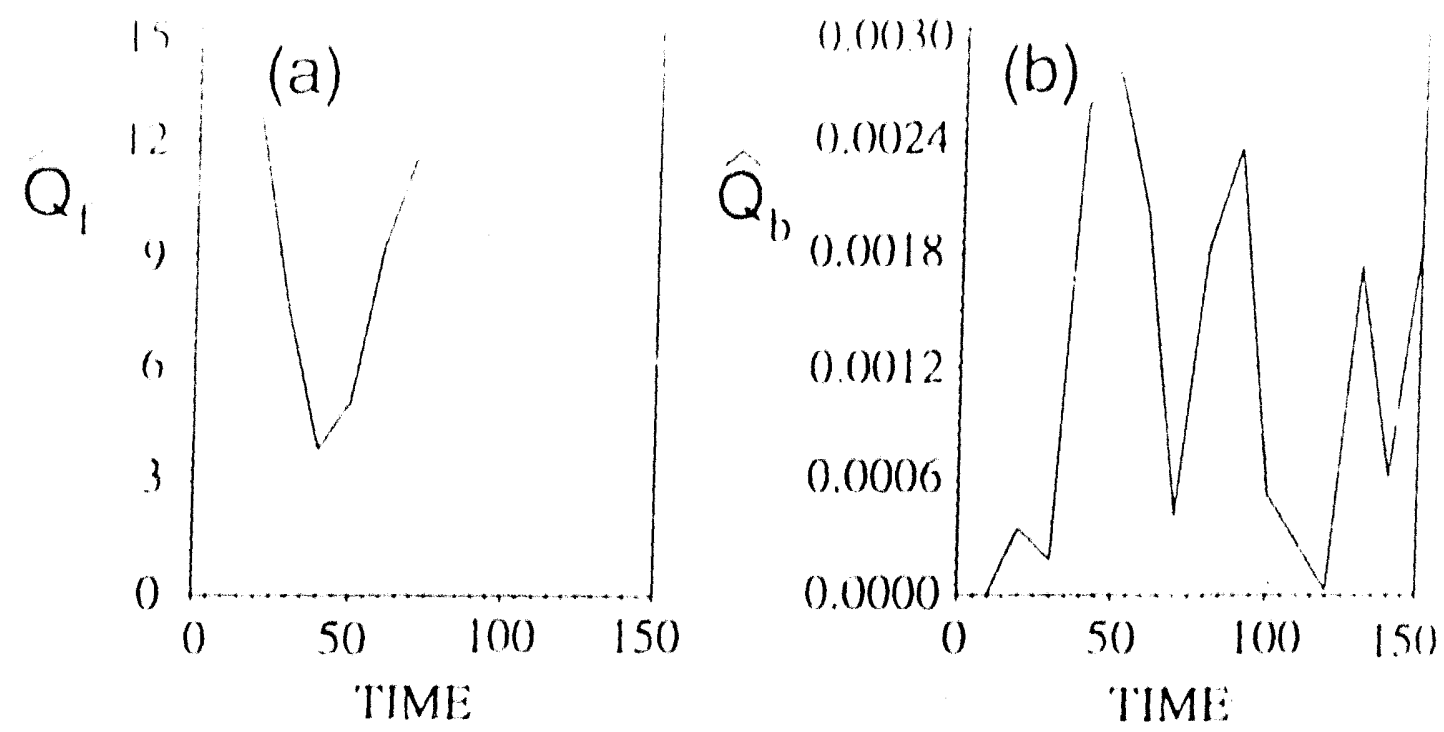

Figure 8: Temporal dependence of electromagnetic emission from the plasma slah) $Q \quad Q(0, t) /\left(n_{\mathrm{ic}} m_{\mathrm{i}} c_{s}^{2} c / k_{0}\right)$ in straight forward $(\theta-0)$, (a) and backward (0) $\left.\pi\right)$, (b) directions. The parameters of this run correspond to curve 4 in figig. 7 .

to the filamentation instability of two cotuter propagating light beans incident and backscattered |31|. This instability, in contrary to the conventional filamentation, is an absolute one and can operate even for the small beam overlapping region if the beam's intensities are comparable. The time history of the far field distribution shows that this absolute filamentation instability arises after the saturation of back ward SBSS and develops in a longer time scale.

Runs with higher lon acoustic damping $\Gamma=0.3$, curve $4 \mathrm{in}$ Fig. 7 , show less reflectivity and more complicated temporal behavior. The reflectivity pulsation's are characterized by the much longer period than the backward SBS saturation time and they illustrate the competition between SBS and self-focusing. This competition is even more visible if the temporal history of the narrow cone emission has been traced, as it is shown in Fig 8 . The brightness of light emission in straightforward $(\theta=0)$ and backward $(\theta=\pi)$ directions oscillate with the period, which is approximately equal to the time the ton acoustic wave needs to pass the incident beam cross-section. This is very clear indication of the inter. action between backward SBS and self-focusing in tabilities. We should note here that the competition between self-focusing and backward SBS was reported recently in Rof [32) in the paraxial optics approximation. In their example the backward SIBS restricts self-focusing instabllity and results in the periodic flashes of backscattering. In our sim. ulations we see a different scenario: SBS has no serious eflect on self focusing saturation, but mainly follows the electromagnetic field intensity variations inside the plasma. This situation is illustrated in Fig. 9, which corresponds to the large value of self focusing parameter $\alpha_{s f}=16$. At time $h_{0} c_{s} t=30$, due to self-focusing high intensity electric field has been generated and boosts backscattering reflectivity more than two orders of magnitude with respect to convective saturated level. Later, the focus position moves forward along. $x$-directon and a new focus starts to appear. This dynamic: belaviot of self focusing in- 


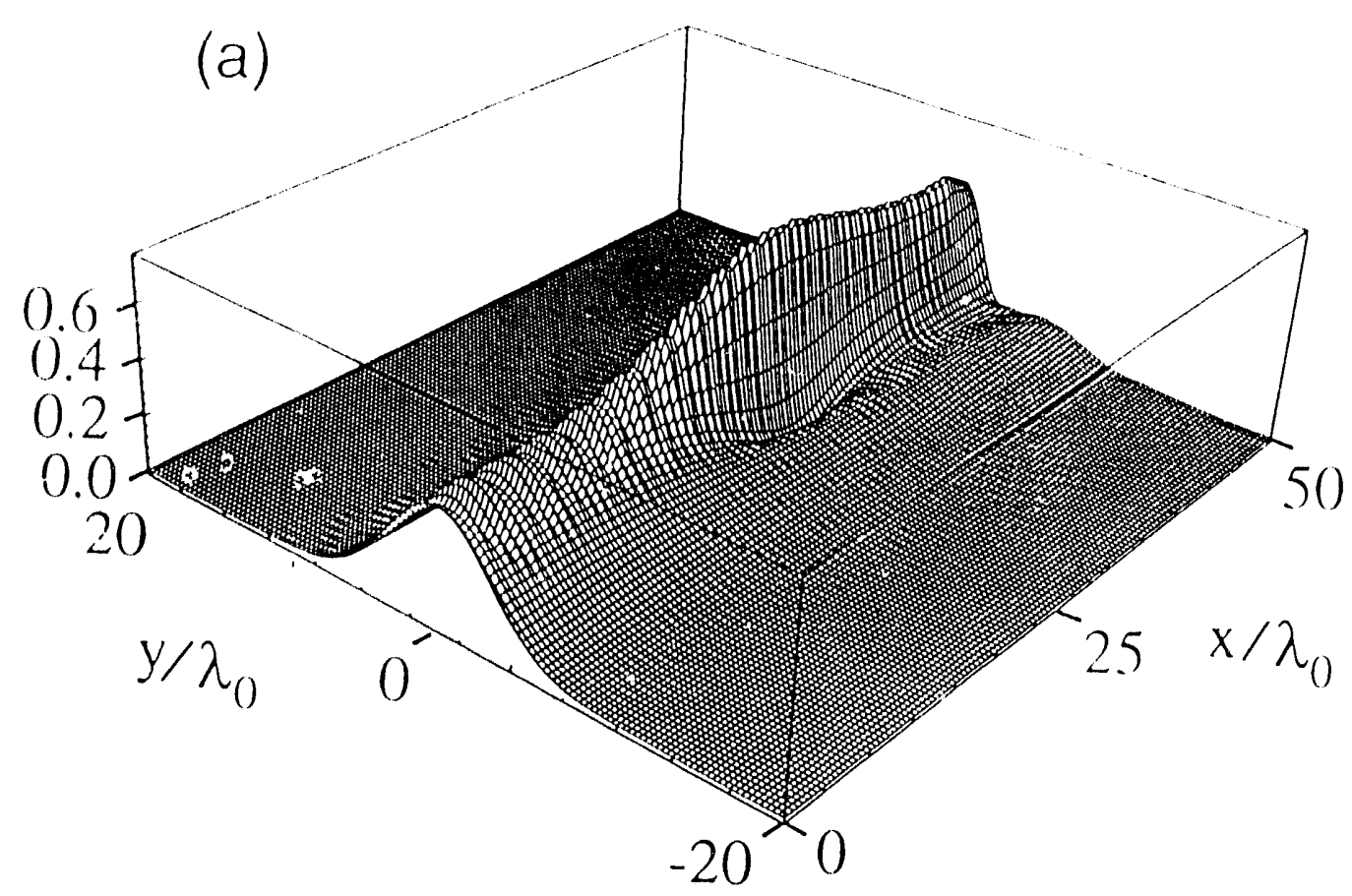

(b)

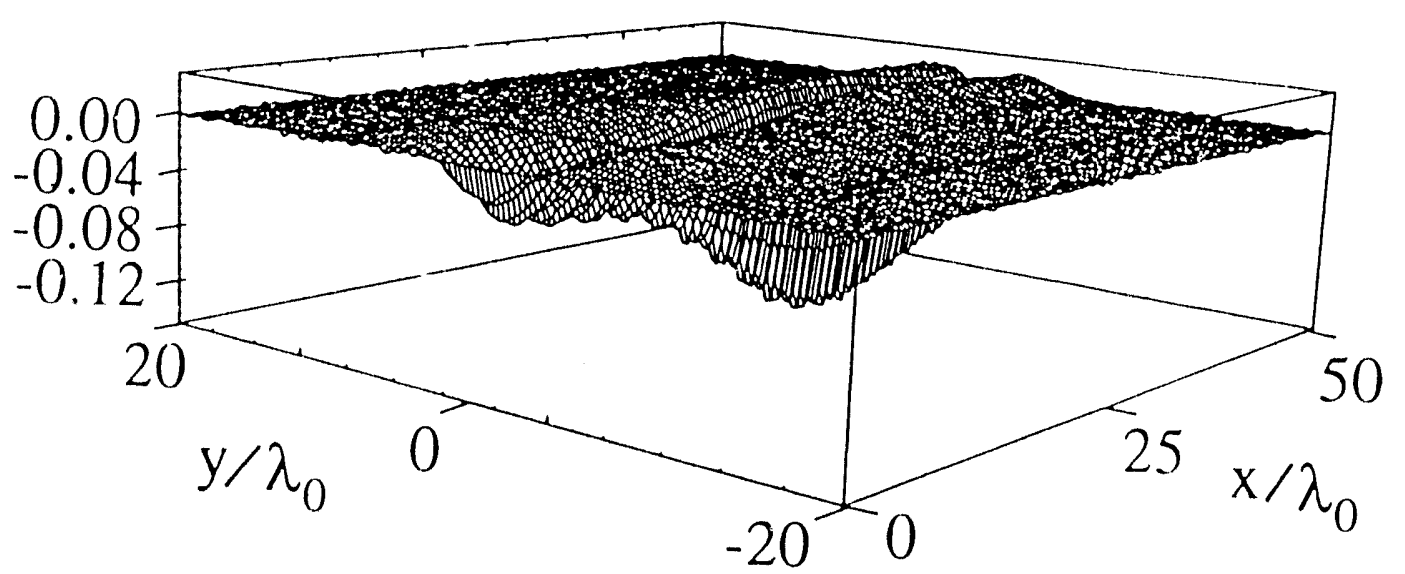

Figure 9: Spatial distribution of the electric field amplitude $|E| / \sqrt{4 \pi n_{i c} m_{i} c_{s}^{2}}$ (a) and the ion density fluctuations $\delta N(b)$ in the plasma slab at time $k_{0} c_{s} t=30$ for the following parameters: $\left|E_{0}\right|^{2} / 8 \pi n_{e c} T_{e}=0.08, n_{i} / n_{i c}=0.5, k_{0} a=20$; interaction length $L=45.5 \lambda_{0}$. 


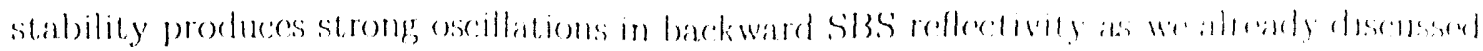
earlier

Comments on 2I) studies on ion wave parametric instabilitios

Two dimensional simulations of laser interaction with ion plasma medes demonstrate a variety of regimes depending on the ion acoustic: wave damping, geometry of the inter action region and the laser beam intensity. There are two main dimensionless parameters which control the interaction processes. The first one is the backward sibs gain coefficient $G_{b}$ arid the second one is the self focusing parameter $\alpha_{s f}$. In the case of small $\alpha_{s f}<1$. the competition between backward, side, and forward Slis takes place The backscat tering unavoidably dominates for the gain $C_{b} \geq 10-12$. For lower gains, forward Sist grows to a higher level due to the saturation of the backscattered SBSS. The characteristic angle of forward scattering is directly related to the smallest spatial scale in the incident. beam cross section. In the long term evolution, signatures of phase conjugation has been observed in the backscattered light signal.

In the case of large values of the parameter $\alpha_{s f} \geq 10$, the main issue is the competition between self- focusing and backward SBS. If the gain coefficient is sufficiently large $G_{b}>$ 15, SBS definitely wins, producing strong pump depletion and arresting self focusing, For smaller values of $G_{b}$, backscattering cannot prevent self focusing, and the latter has a dramatic effect on SBS temporal evolution. This regime is characterized by strong temporal oscillations of the backscattered and transmitted light and production of high amplitude electric field spikes inside the interaction region. The field maxima have a short life-time, they dissolve, and grow again in a different location. This behavior has a lot in common with the "flicker" mode of self-focusing reported in Ref. $|33|$ with the difference that in our case it happens even for a smooth Gaussian incident beam and backscattering plays an important role in the evolution of the instability. The transmitted light in that case is splitted into few beamlets with time varying intensities and propagation directions.

This study has been restricted here to the case of a smooth incident beam and a homogeneous plasma slab. This has been made with the idea to elucidate the characteristic regimes of the interaction, and provide a simple analytical description of any of the processes. The more realistic models of strong amplitude and/or phase modulated incident electric field which are relevant to the experiments with RPP, ISI and SSD laser beams (21) are now under investigation. But as a first order approximation, the present paper may be regarded as the study of temporal behavior of a one speckle of a multimode laser beam in a plasma. From that point of view, the control parameters $G_{b}$ and $\alpha_{s f}$ can be used for the description of multimode beams also, if the corresponding parameters will be evaluated to any speckle or "hot spot" of the laser beam.

\section{SCATTERING INSTABILITIES IN SHORT TIME SCALES; EXPERIMENTAL EVIDENCE}

In this section we will review results from experiments performed with a laser pulse of $600 \mathrm{ps}$ duration, and in which the temporal resolution of the diagnostics was sufficient to make evident many features occurring in time scales of the order of ten picoseconds.

\section{Experimental Configuration}

Preformed plasmas experiments were developed at Ecole Polytechnique to investigate laser-plasma interactions in long and quasi-homogeneous plasmas [34]. These experiments were performed using two beams of the LULI Nd:glass laser facility [35|. The approach 


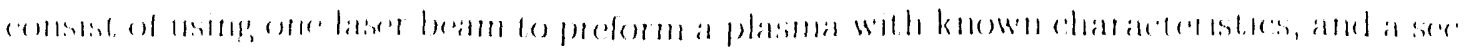

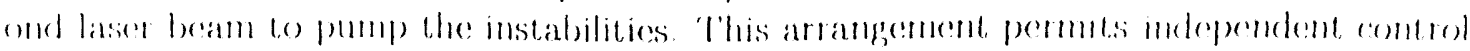

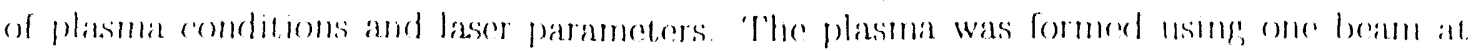

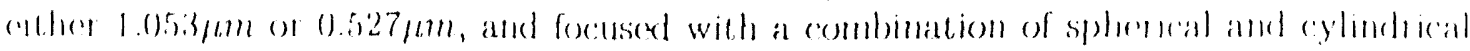

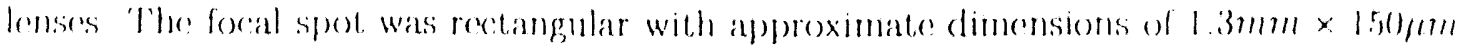
The haser pulse was (anussian in time with a full width at half maximum of 600 ps, with no temporal modulation. The incident intensities on target was $1-2 \times 10^{13} \mathrm{~W}^{1} / \mathrm{cm}^{2}$. The targets were (CH foils with thickness between $2007 \mathrm{~m}$ and $800 \mathrm{~nm}$. 'To avoid extge effects, the targets were approximately $700 / \mathrm{m}$ wide, smaller than the length of the focal spot The intial foil thekness and time delay between the two beams determines the plasima parameters at the time of the interaction Plasmas with $n_{c} / n_{c}<0.2$ and $T_{e}<0.5 k$ b were obtamed 600 ps after the peak of the pulse [36|. The second beam (either at the fundamental frequency or converted to second harmonic) was focused with an $1 / 2.7$ lens along the long axis of the plasma. The focal spot diameter was 70 / energy, with a maximum averaged mitensities of $2 \times 10^{15} \mathrm{~W} / \mathrm{cm}^{2}$. Refraction effects from the interaction beam wore negligible. The length of the interaction region ( 2300 pm) was set by the focal depth of the focusing lens and not by the plasma length.

A diagnostic for the plasma electron density is provided by the SRS light itself. From the linear dispersion relations and the wave-matching conditions for the Raman process. there is a umique correspondence between the plasma density $\left(n_{e} / n_{r}\right)$ where the Raman light originates, and the scattered wavelength $\left(\lambda_{s}\right)$. For a low plasma donsity this is given by

$$
\begin{gathered}
\frac{n_{e}}{n_{c}}=\left(1-\lambda_{o} / \lambda_{s}\right)^{2}\left|1-5.9 \times 10^{-3} T_{c} \int(x)\right| \\
f(x)=\frac{(1+x)^{2}}{(1-x)^{-2}}\left(\frac{1}{x}\right)\left(3 x-x^{2}-1\right)
\end{gathered}
$$

where $x=\lambda_{o} / \lambda_{s}$ and $T_{e}$ is the electron temperature in $\mathrm{keV}$.

\section{Stimulated Raman Scattering}

Stimulated Raman Scattering (SRS) was studied both in backward and in forward directions. The backward Raman emission was collected by the main focusing lens, while forward Raman was collected by a second $\mathrm{f} / 2.7$ lens in the forward direction. Typical timeresolved spectra for both forward and backward emission, recorded from the same shot, are shown in Fig. 10. The temporal resolution is determined by the combination of the resolution of the streak camera and the temporal dispersion introduced by the diffraction grating spectrometer.

They both show a narrow spectral emission corresponding to a narrow range of plasma densities present in the interaction region. The Raman spectra corresponds to an electron densities $\sim 0.05 n_{c}$ at the beginning of the pulse, and shifts later in time towards lower densities, down to $0.03 n_{c}$ before the end of the pulse. At these low clectron densities, Landau darnping of the electron plasma waves quenches the SRS instability. The lowest electron density at which backward Raman light is observed is referred as the Landau cut-off and, because of its dependence on electron temperature, it is often used as a temperature diagnostic. For the experiments described here, the cut-off corresponds to an electron temperature of $0.4 \mathrm{keV}$. The mean electron density deduced from backward and forward Raman spectra were in agreement for different time delay between the beams and for different target thickness.

Both backward and forward Raman spectra exhibit temporal structures which occur in burst lasting < 20ps. Similar temporal structures are observed in the SBS light, which will ba discussed in the next section. Although the streak carneras did not have an absolute 


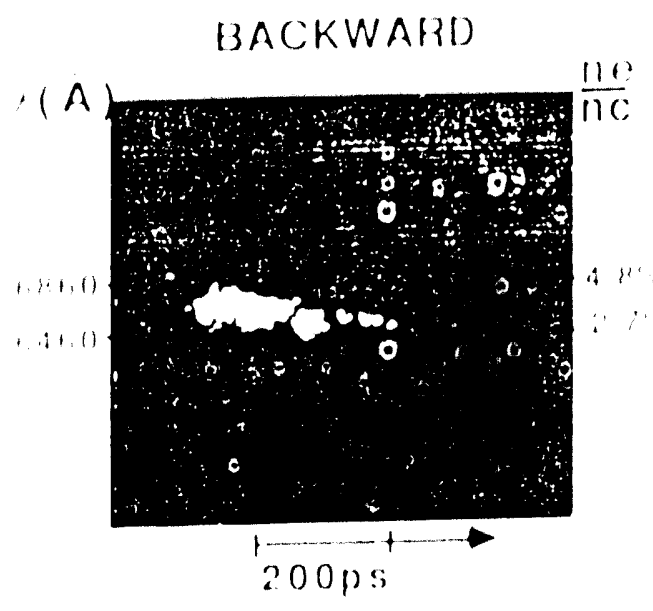

Relative Time

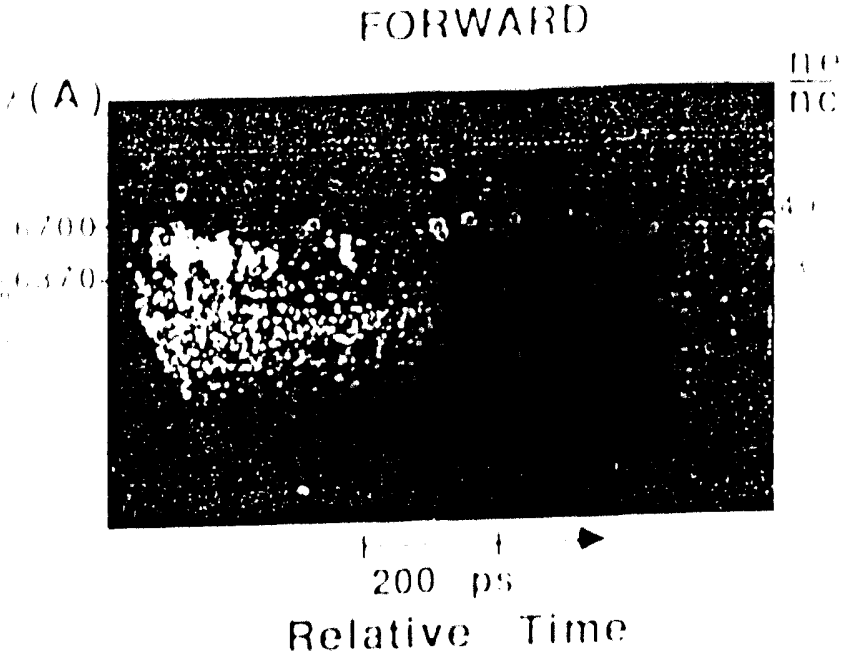

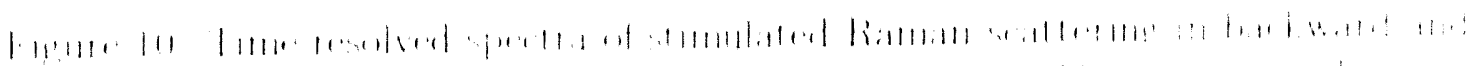

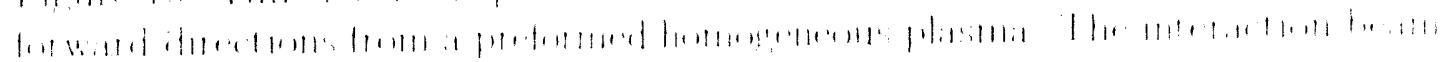

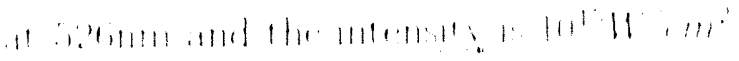

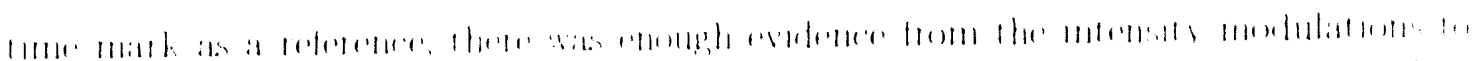

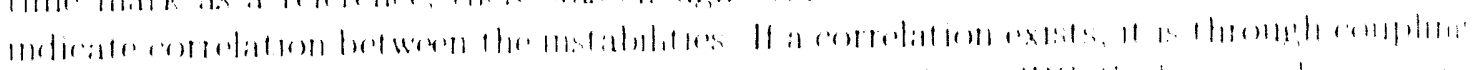
of 1101 :

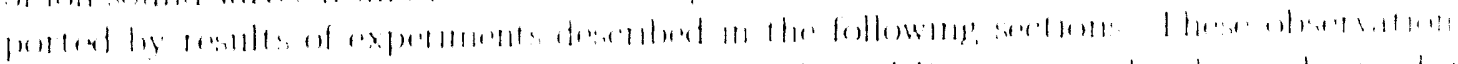

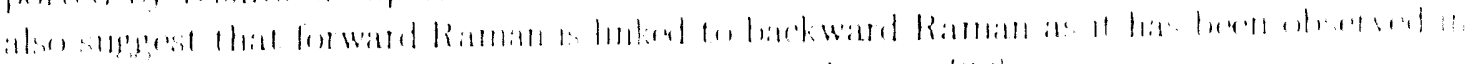

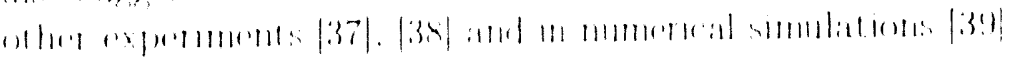

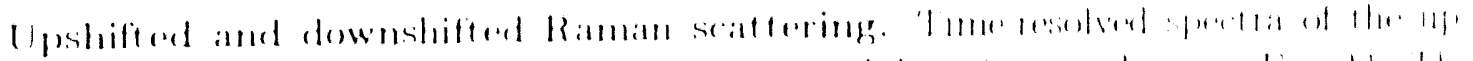

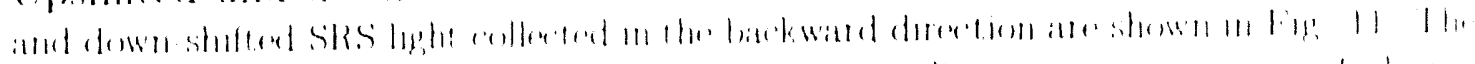

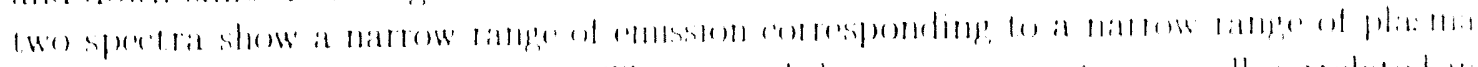

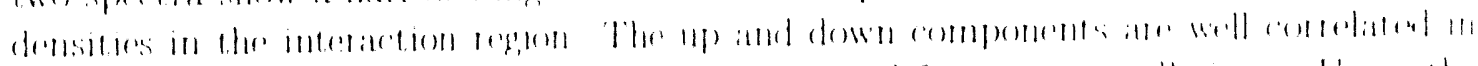

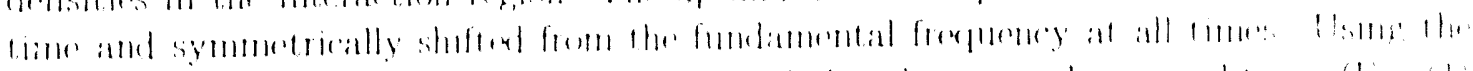

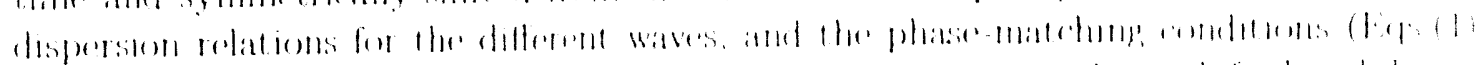

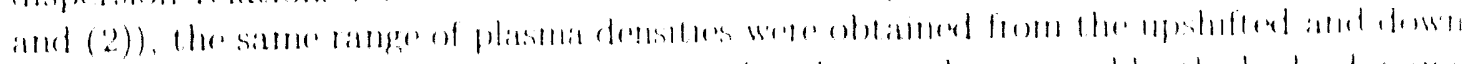

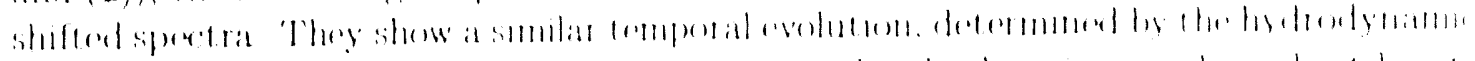

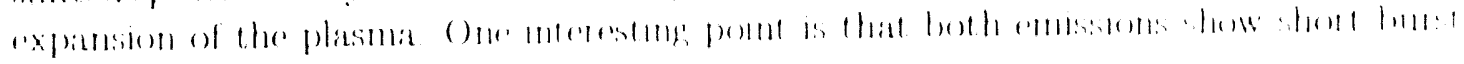

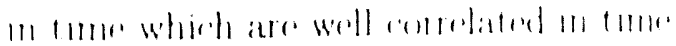

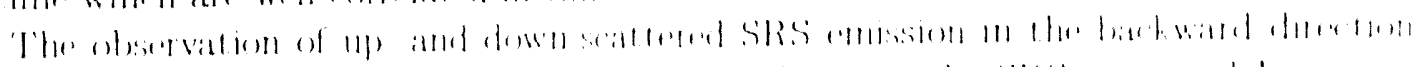

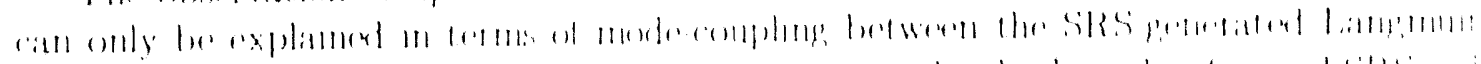

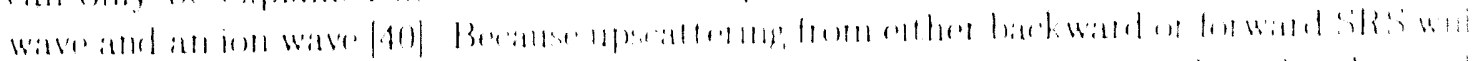

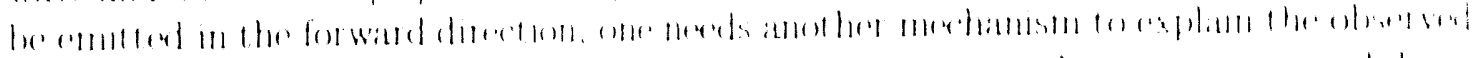

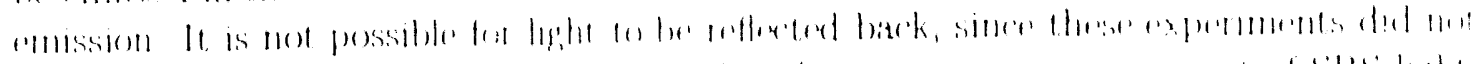

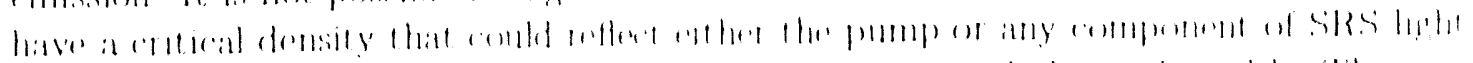

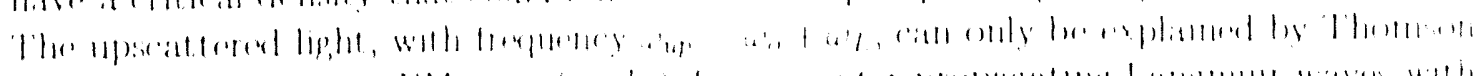

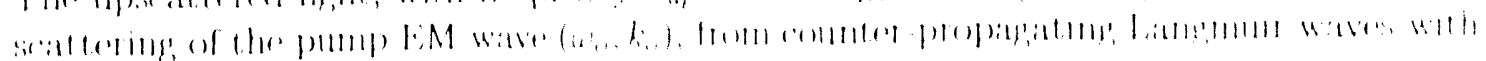




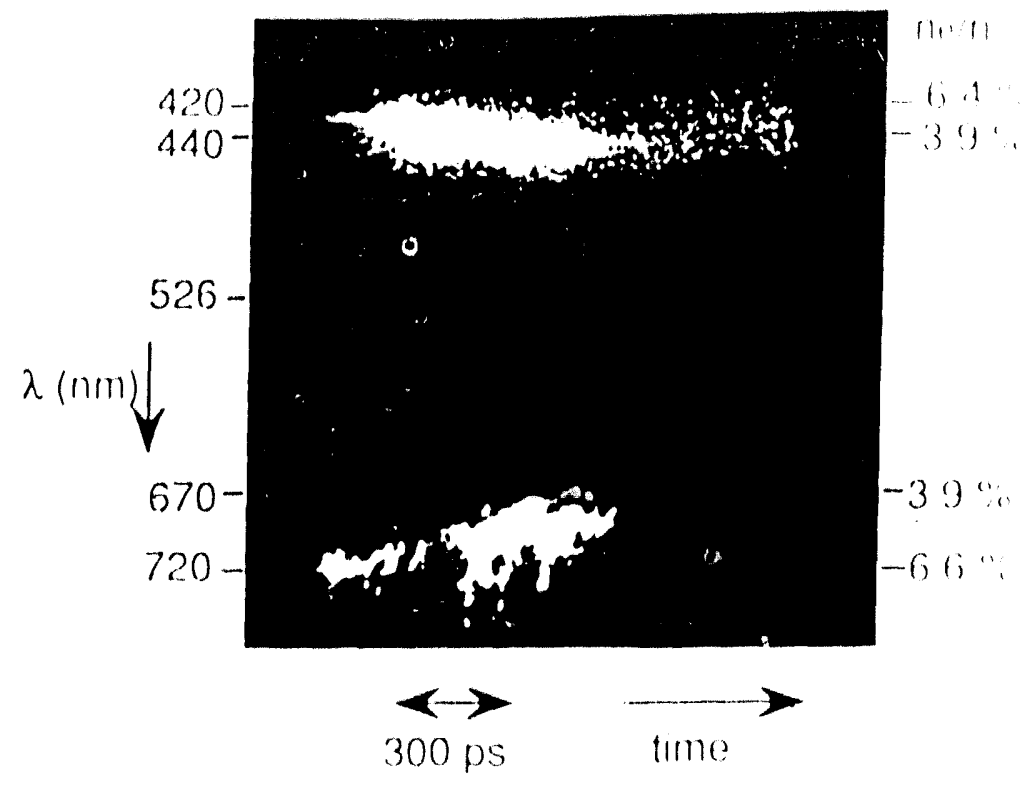

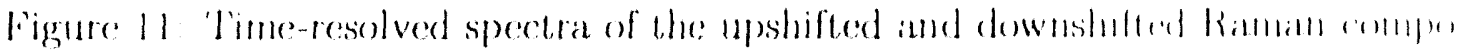

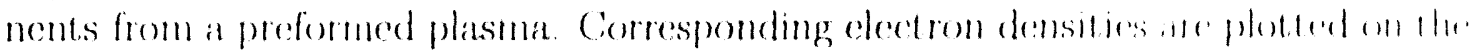
right-hiund scale.

almost the same frequency and opposite wave veetor $\left(k_{u p} \quad k_{s}\right.$ shs $\left.2 k_{0}\right)$ than the sks driven langmuir wave. Several meehanisms have been proposect for the origin of a commen propagating electron plasma wave. The correlation between the upshefted stes leght and the SBS backscattered observed in two different plasma configurations (preformed plitsinia and thin target foils) supports the conjecture of mode coupling through the Siss drowen ion sound wave. This coupling could be responsible for the temporal stuctures that have been observed in both up- and downshifted time-rosolved spectra

\section{Stimulated Brillouin Scattering}

Backscattered Stimulated Brillouin Scattering emission was studied usmg a similat op. tical arrangement as described in the previous section. The spectra were recorded using, it combination of high resolution spectrometer and streak camera, with a wivelength reso. lution of $0.01 \mathrm{~nm}$ and a temporal resolution of $30 \mathrm{ps}$. The plasma was imaged onto the slu. of the spectrometer, thus allowing some spatial resolution. This permitud to collect Bril. louin light coming only from a small region in the center of the plastma. This is differemt from other experiments where light coming from a larger volume of plasma is collexterl on the spectrometer, leading to temporal smoothing of the spectra due to spatial integration Since the plasma was under-eritical at the time of arrival of the interation beam, the backreflected light was due to SBS, with no contribution from specular teflection

A time-resolved spectrum is shown in Fig. 12. The spectrum is very narrow at all tumes and red shifted from the laser wavelength by a constant value of around $0.7 \mathrm{~nm}$. 'These spectral features confirm that SBS is emitted from an homogeneous plasma and with very little flow velocity along the laser axis. The spectra is completely different from those observed in thin exploded foil experiments, where red shifts increasing as a function of 


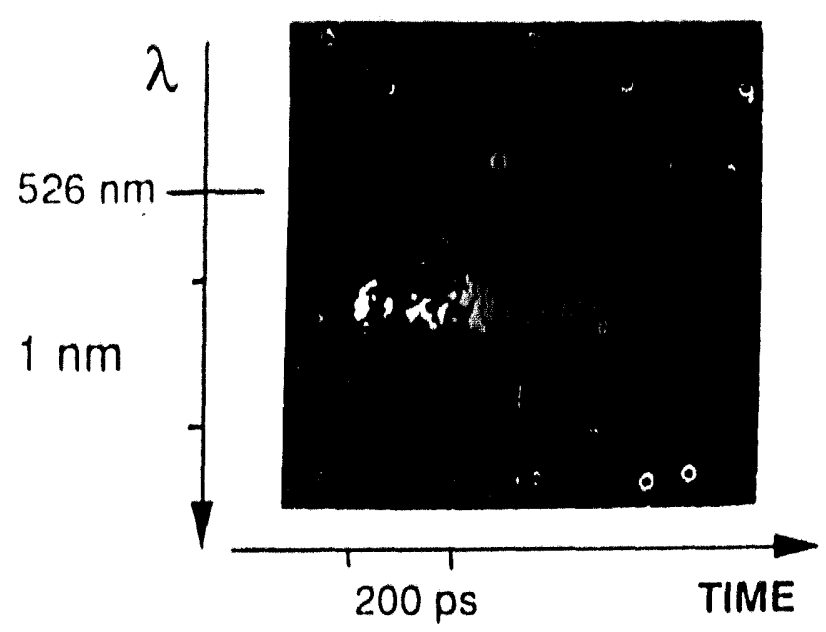

Fignere 12 T'ime-resolved spectrum of stimulated Brillonin batekscatterims, Lrom in

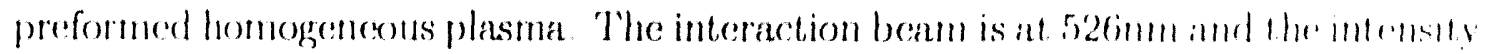
is $10^{15} 1 \mathrm{~W} /(\mathrm{m})^{2}$

time are attributed to Doppler effect from the recoiling foil. The combination of a long interaction region and low flow velocity along the laser axis explains the high level of sibs reflectivity observed in this experiment, as compared to exploding foil targets, which have an intrinsic larger velocity gradients and lower reflectivities. Under similar irradiation conditions, time-integrated reflectivity's were of the order of $10^{-2}$ in preformmel plasmas. and around $10^{-4}$ in thin exploded foils.

A feature we want to underline in this review is the fast evolution of observer re. flectivity. SBS emission appears as a collection of very short bursts, shorter than 30ps, indicating the rapid growth, saturation and quenching of the instability This temporal modulation do not show any periodicity and vary randomly from shot to shot. Instanta. neous reflectivity's are then much higher than time-integrated reflectivity's, demonstrating the difficulty in comparing experimental data and theoretical modeling. These modula. tions are not coming from the incident laser pulse, and are evidence of non linear effects on the ion waves excited by the instability. One possible hypothesis for these structures is the coupling of ion sound waves due to Brillouin and electron plasma waves due to Raman |41|. Stimulated Raman emission recorded during this same experiment showerl similar temporal behavior.

\section{Filamentation}

In laser produced plasmas, filamentation is a difficult instability to diagnose. All experimental evidence of filamentation have been inferred from features observed indirectly. In the experiment described here, evidence of flamentation was found from signatures observed in the transmitted laser light, in Ramaic emission, and in second harmonic emission. The observed temporal structures indicate the presence of density chamnels in the 

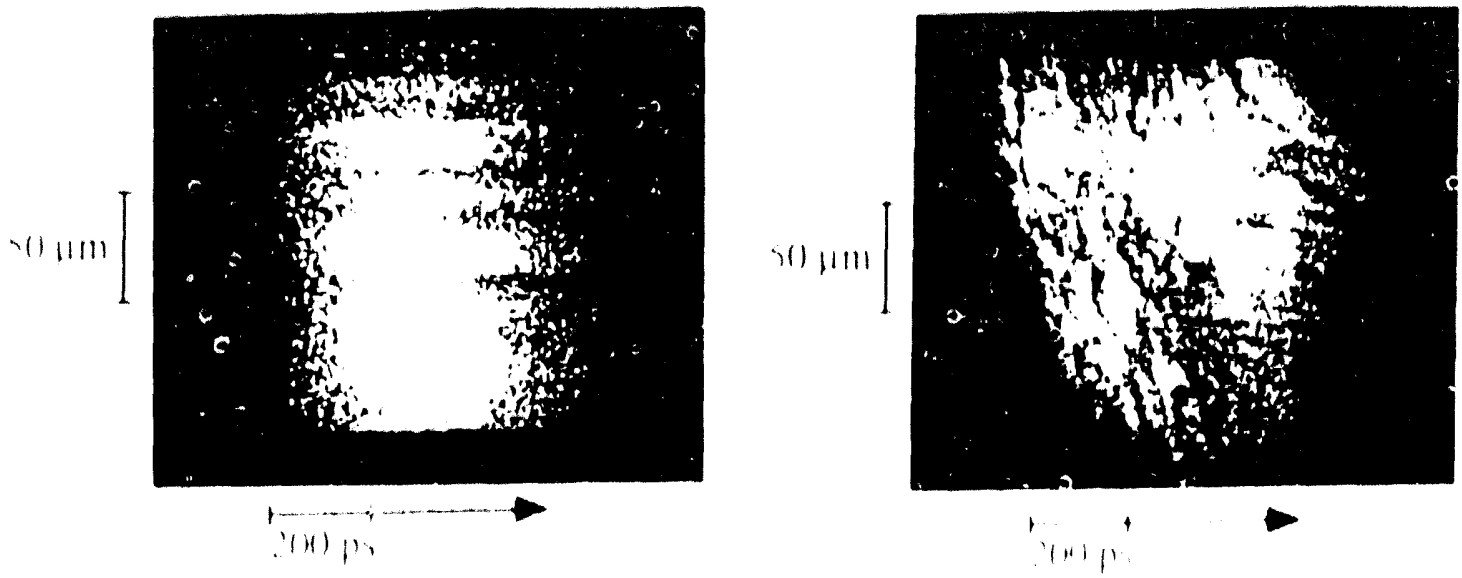

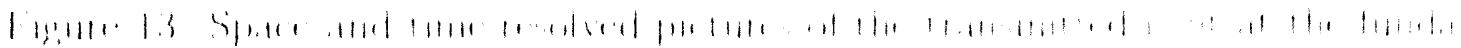

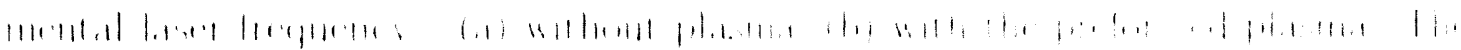

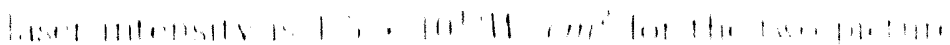

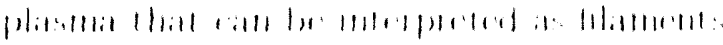

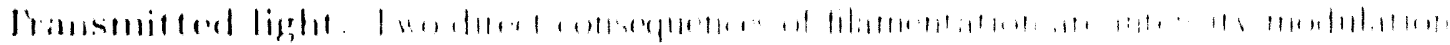

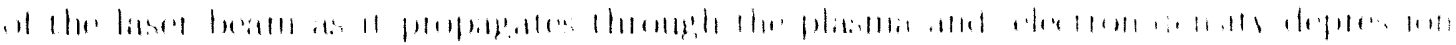

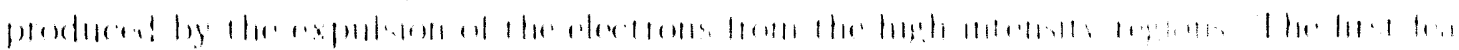

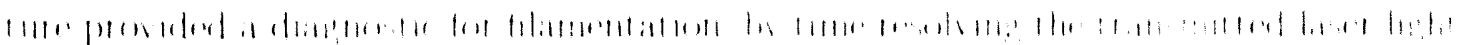

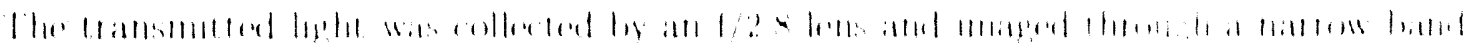

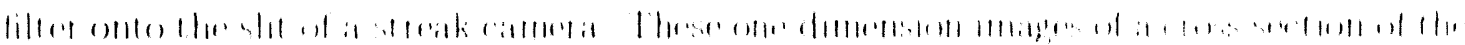

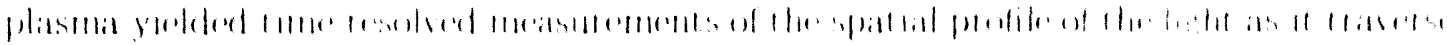

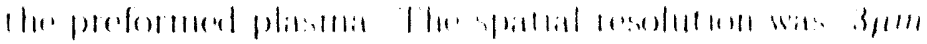

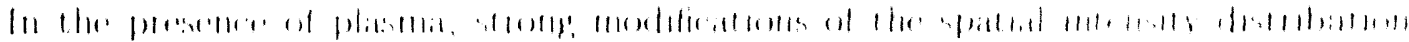

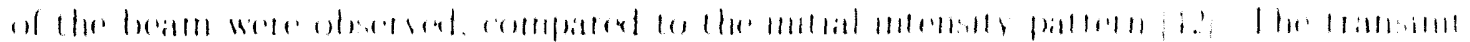

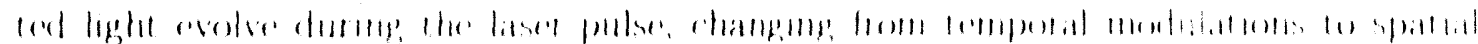

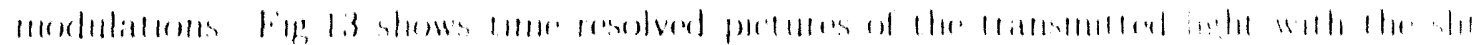

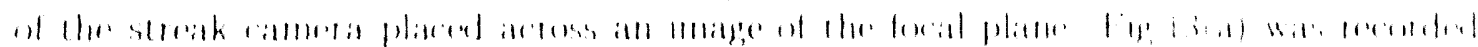

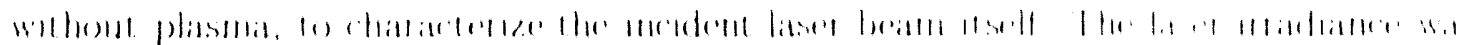

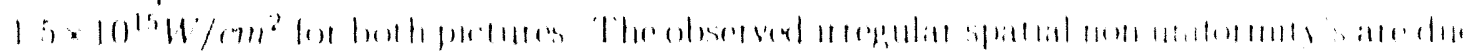

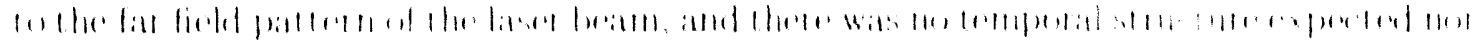

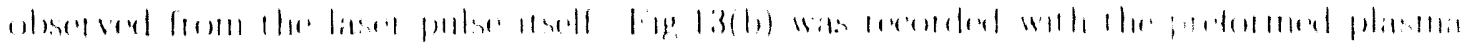

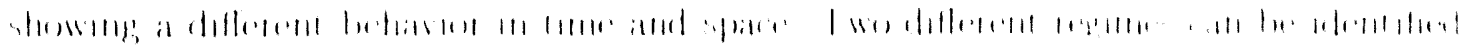

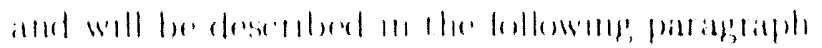

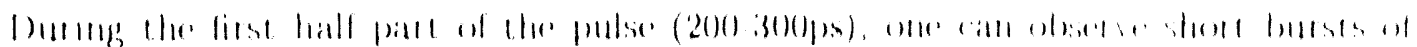

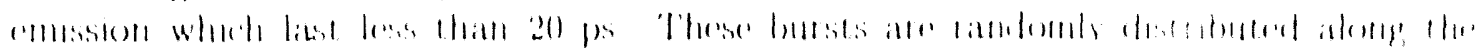

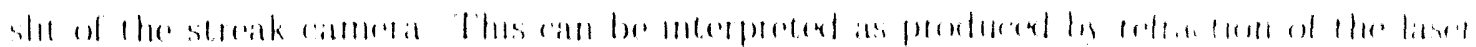

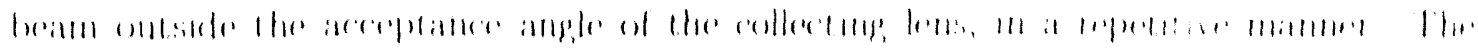




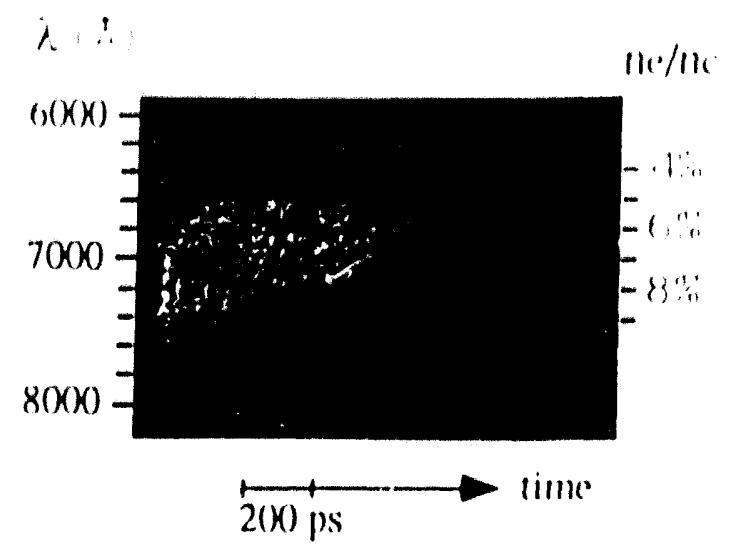

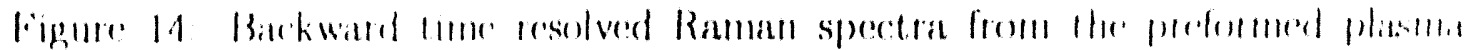
showing Raman light emilted from the background density and Rambln growemgen

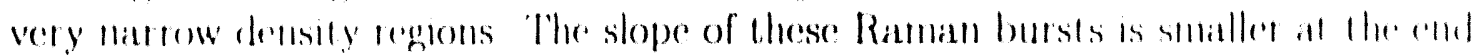
of the pulse than at the begimning of the pulse. The laser wavelengeth is 52 intur and the nominal laser intensity is $1.5 \times 10^{15} \mathrm{~W} / \mathrm{cm}^{2}$

sudden refraction of the beam could be explained by a fast change of the phasma dertsuly: as expected from the nonlinear evolution of filaments inside the plasma.

The later quart of the pulse shows spatial structures which are relatively stable in space and time, indicating that the laser beam was divided into small beamlets. pessible. due to s:mall plasma density chamnels. The size of these channels are typically of the order of 5 to $10 \mathrm{am}$, corresponding to regions of lower plasmn density, as one would encounter in filamentation. Some of these features have been observed in mumerical simulations of the nonlinear evolution of filamentation, although the two regimes observed early and late in the laser pulse are not clearly understood.

Raman spectra. An example of time resolved Raman spectra is shown in Fig. 14. The background Raman emission displays the characteristics which have been discussed above Superimposed to this emission one can observe structures with temporal evolution which interpretation requires a different mechanism. They exhibit a temporal slope which is different from the mean slope of the spectrum. They indicate that Raman light is emitted from very narrow density channels with an electron density that is decreasing in time This can be interpreted as if the Raman instabllity is growing in filaments induced by hot spots due to the laser intensity distribution. The Raman emission follows the electron density evolution inside the filament, as indicated by the shift towards shorter wavelength in time. The slope of each of these narrow structures is larger than the mean slope of the spectrum, showing a faster decrease of the density in these regions. This temporal evolution is different at the beginning and at the end of the laser pulse. The slopes in dicate faster density evolution in the beginning of the laser pulse, consistently with the rapidly varying modulations observed in the transmitted images. Different theories $|4.3|$ predict modifications of Raman growth rates in filaments. These theories have been usey to explain Raman emission with laser intensities below threshold, as well as to explain structures observed in the Raman spectra. In this experiment the laser intensity is above the Raman threshold, so there is no need to invoke filamentation to explain the Raman. background emission. However, the very narrow structures observed in the spectra are 


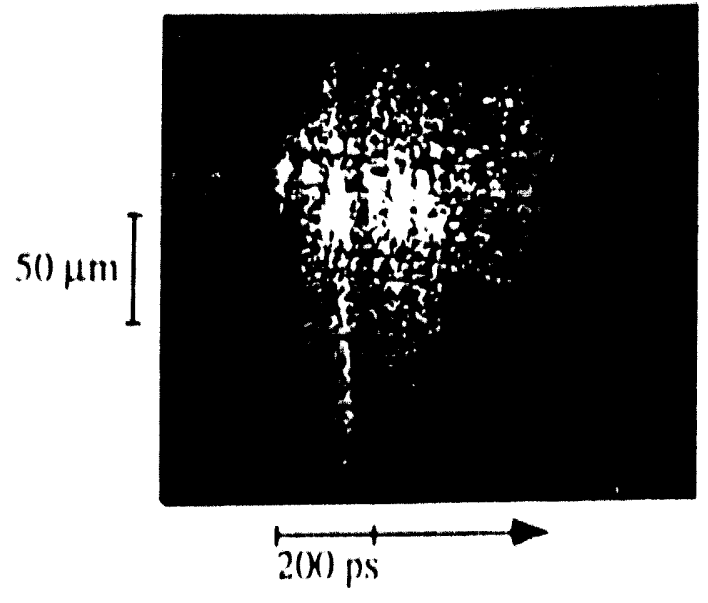

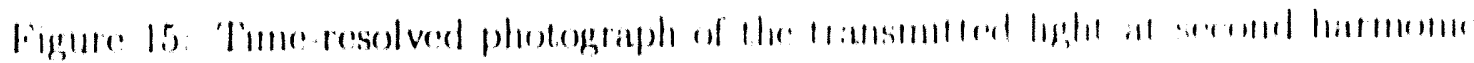
of the fundamental laser freculency

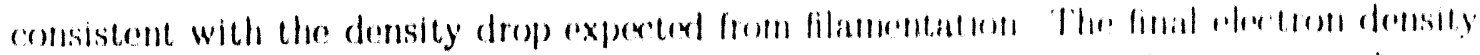

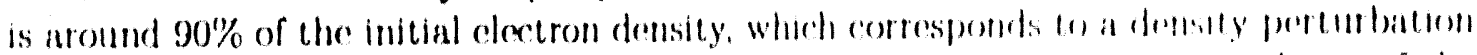
produced by filamentation of $d n / n-10 \%$, in agerement with the theremetial stze of the density fluctuation given by $|41|$

$$
d n / n=1-e x p\left|-0.375\left(v_{o} / v_{t h}\right) 2\right|
$$

where $v_{0}$ is the electron quiver velocity and $v_{t h}$ is the olecton thermal velecity . The time noeded to modify the electron density varies from a fow picosireonds at the beginning of the pulse to $\sim 100 \mathrm{ps}$ at the end of the pulse.

Second harmonic emission. Second harmonic emission was collexted in the forward direction, using a $1.053 \mu \mathrm{m}$ interaction laser beam. A two dimensional crosis section of the interaction region was imaged with a combination of two lenses into film with a total magnification of 35 . A one dimensional strip actoss the interaction region was imagerl into the entrance of a streak camera, thus providing a time resolved record A time-resolved photograph of the transmilted light at the second harmonic is shown in lig. 15.

The width of the streak camera slit corresponded to about $150 / 2 \mathrm{~m}$ in the plasma. The emission is observed to have strong temporal and spatial modulation. The observerd short temporal bursts have a duration of the order of $20 \mathrm{ps}$, with a spacing between bursts of a few tens of picoseconds. The spatial structures have a dumension between 6 and $10 / \mathrm{mm}$ The laser energy converted into second harmonic emission, and collecterl into the $f / 2.5$ lens, was $3-6 \times 10^{-6}$.

Generation of second harmonic emission in the forward direction in underdense plasma requires the presence of a radial modification of the plasma density in order to introduce a strong electron density gradient perpendicular to the beam direction. The filamentation of the laser beam propagating in the underdense plasma produces transverse density gradients, which in the presence of the strong laser electric field, give rise to localized electron currents at $2 \omega_{o}$. The struccures observed in time-integrated images show emissions which are well defined in space and which can correspond to filaments. 


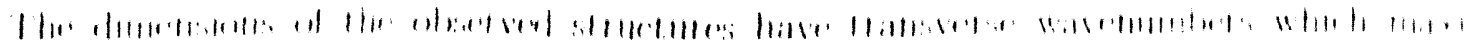

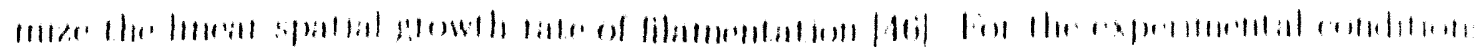

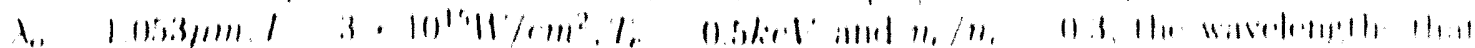

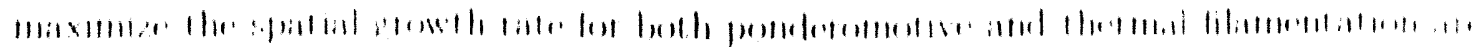
1, $1, \quad 2$ :

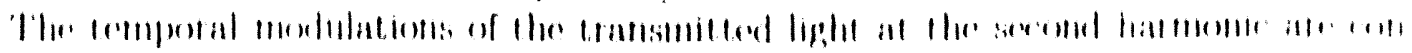

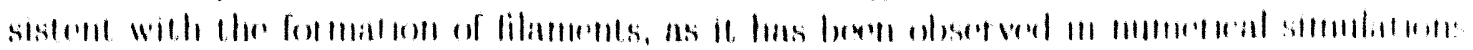

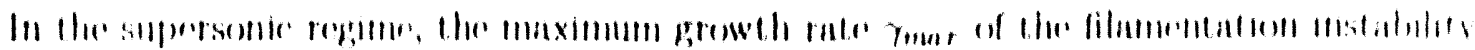

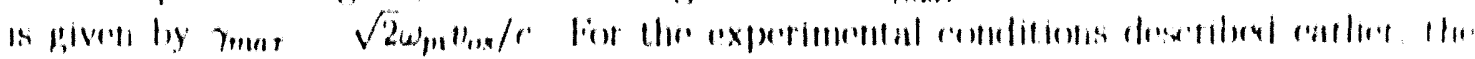

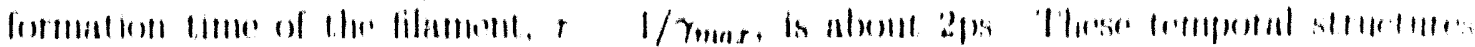

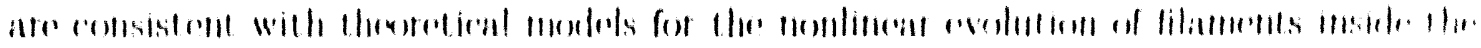
phasmin

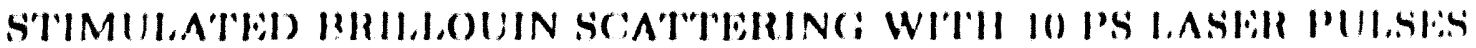

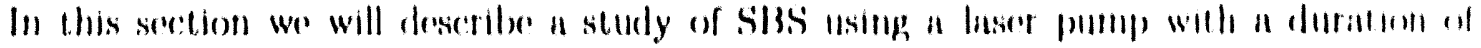

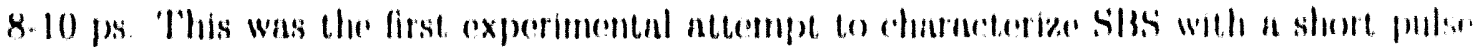

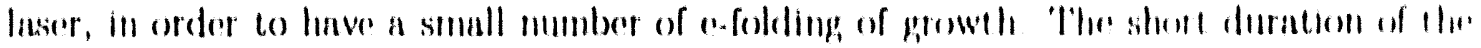

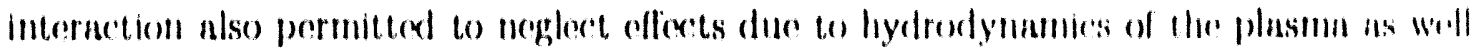

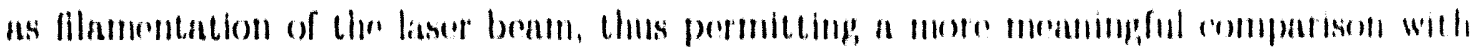

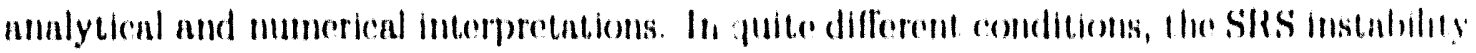
has bern studical with a ps laser pulse, both experimentally |49), and thererotically find

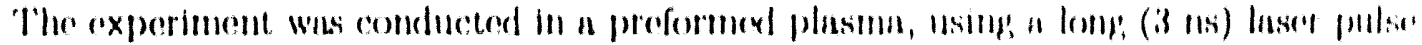

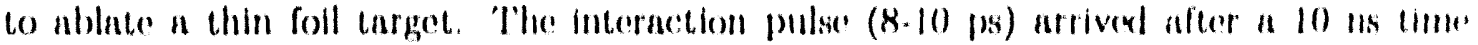
dolay to eliminate any coupllang with SBSS fon waves produced by the lirst lased pulse $A$ preformed plasma has the advantage of providing a longer density sealo lenghth than what would be obtained with the sliort pulse alone, as woll as providing p'asma conditions which are independent of the short pulse intensily. The time integnateni reflectivity of the Silss seatcered light was recorded will an $S-1$ photommltiplier, while 1 detector arrey providesl a time integrated spectra. The photomultiplier have sullicient sensitivity to mensure low levels of SBBS, as well as the EM noise level of the plasma, with a temporal resolution sufficient to separate the SiBS emission of the short pulse from that of the long pulse.

The short pulse was generated from a 100 ps mode-locked pulse, and then chirperd and dispersed in a $2 \mathrm{~km}$ long optical fiber. At the output of the opetical fiber the pulse had a spectral width of approximately $2.5 \mathrm{~nm}$ and a duration of $300 \mathrm{ps}$. This was then amplified in a $Y \wedge$ G regenerative amplifier to a pulse energy of a few $m$. Due to the marrow gain bandwidth of YAG (0.2-0.3 nm), only the central part of the pulse was amplifled, giving an output pulse with a duration between 8 and $10 \mathrm{ps}$, and a near transform limited spectral width. This novel technique permitted to obtaln short pulses $(8-10 \mathrm{ps})$ at TW power levels, wilhout having to use pulse compression schemes with diftraction gratings, as is normally used in standard chirped pulse amplification (CPA) systems. The pulse was then amplified using a combination of rod and dise gilass amplifiors with a maximum aperture of $10 \mathrm{~cm}$. The maximum energy of the short pulse was 10 . The pulse durntion was measured using a single-pulse autocorrolator. $\wedge$ sepurate nutocorrelator was used to monitor the level of prepulso, which was less than $10^{-1}$ and sufficient for this experiment. The long (3ns) pulse was generated in a separate single longitudinal-mode oselllator, and amplified in a separate amplifior chain to an energy of npproximately $10 \mathrm{~J}$. Both pulses were brought together with opposite polarization so that they could be combined using a polarizer, and then focused on larget with a single $f / 10$ lens.

Plasina parameters desirable for the experiment were obtained by irradiating a thin plastic $1.5 \mathrm{~mm}$ thick foll, with the $3 \mathrm{~ns}$ laser pulse at an intensity of $10^{12} \mathrm{~W} / \mathrm{cm}^{2}, 10 \mathrm{~ns}$ 


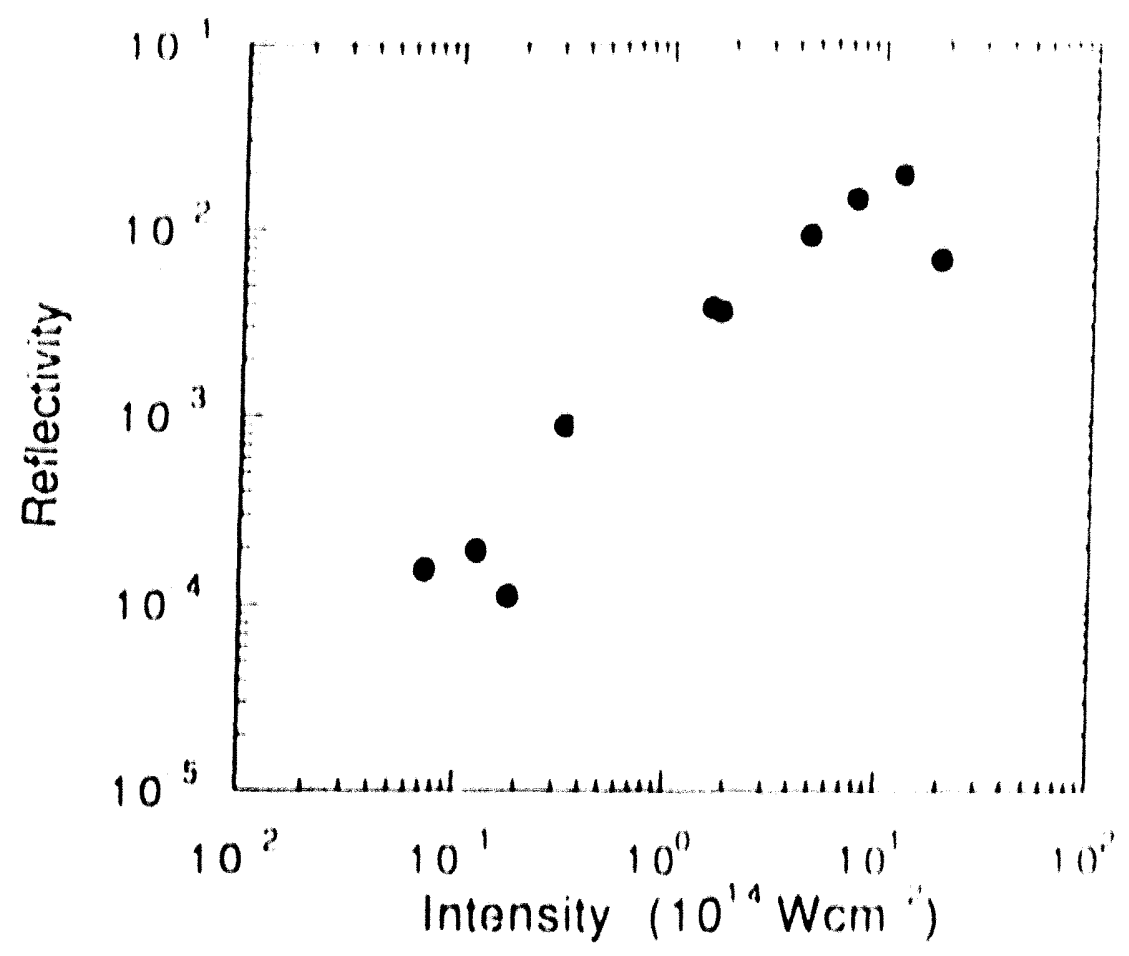

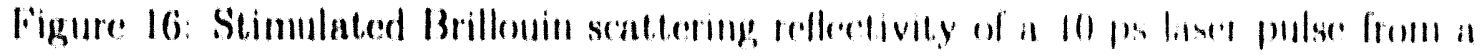
preformed plasma, as a function of the laser intensily

before the arrival of the short pulse. This arrangement produce $"$ plisma with a long electron density scale-length, maximum electron density of approximminly $10 \% n_{12}$, and high electron temperature. The 10 ns delay providexl enough separation betwoen the two pulses to nvold any seceding of SlBS in the short pulse due to Slis produced by the lonk pulse. The plasma temperature at the time of interaction was tetermmend by the intensity of the short pulse. The increase in plasmn temperature was calculated assuming inverse bremsstrahlung absorption, and compared to fluid code simulations for a short pulse intensity of approximately $10^{15} \mathrm{~W} / \mathrm{cm}^{2}$, the temperature at the time of the interaction was approximately $1 \mathrm{keV}$.

The reflectivity of the $10 \mathrm{ps}$ pulse is shown in Fig. 16 as a function of the laser inton. sity. While at low laser intensiltes the reflectivity has a well definer non zero slope, at high intensitles there is clear evidence of saturation. The reflectivity measurements are time integrated, and one expect that the instantaneous reflextivity will be higher at the end of the short pulse. The temporal resolution was sufficient to separate the plasma emission at the the of interaction from any emission or reflection occurring when the plasma forming laser pulse was present. Figure 17 shows the temporally integrated spectrum of the backscalterd light. The SBS contribution from the long pulse was less than $10 \%$ of the component from the short pulse. For low laser intensities $\left(l<10^{14} \mathrm{~W} / \mathrm{cm}^{2}\right)$ the backscattered light consists of two well deflned components: one centered close to the incident laser wavelength, and a sccond component shifted by approximately $0.4 \mathrm{~mm}$ and with a spectral width of approximately $1 \mathrm{~mm}$. The spectral widch of the unshifted component is approximately $0.2 \mathrm{~nm}$, which corresponds to the instrumental resolution. For higher laser inconsitles, the red shifted component has a similar shift, but is wider and becomes the predominant part of the spectrum. At intensities of about $10^{15} / \mathrm{Wcm}^{2}$ a second component is observed which is superimposed on the main component and has a wider spectral width. The observed spectral widths, close to the value of the ion acoustic: frequency, indicate that for all laser intensities the interaction is weakly coupled. In a 

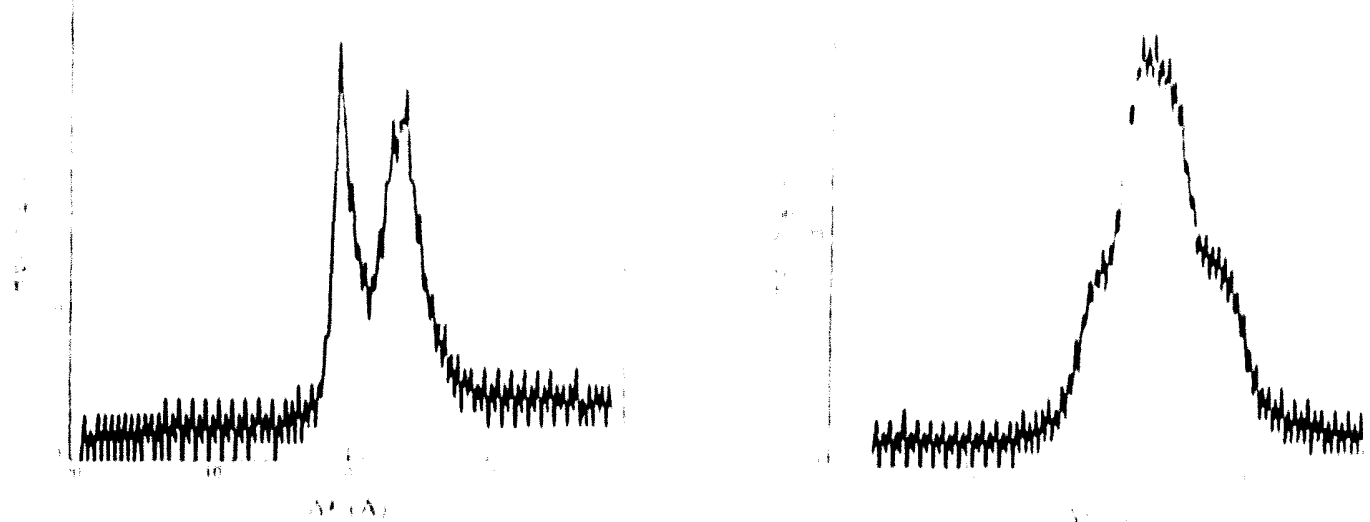

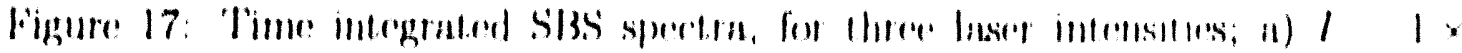

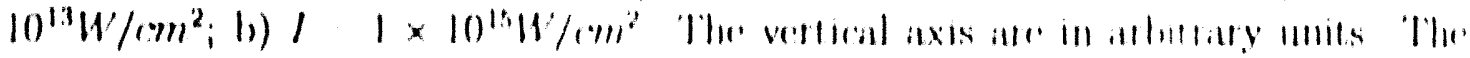

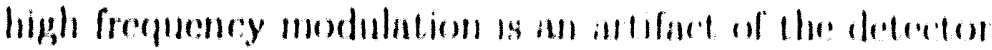

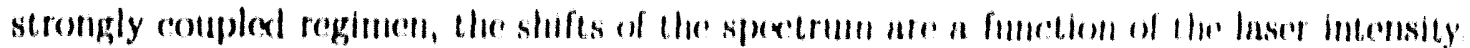

The dependence of the rellextivity on the laser internsity did not aptere with results from 2D) modeling (0| |5|. At low laser intensity, the modol prodicleal roflestivity's two order of magnitude higher than observes experimentally. To explain this diserepancy the nuthors invoked the coupling betweren the Ruman and Brillouin instabilities, a process that was accounted for in the therory

One may conjocture that although sles develops from a lower level of noise, th hus " significantly larger growth rate; the plasma waves can then couple to the fon acous. tic waves, give rise to the parnmetric decay instability which lends to cascadit g and/or collepse. These nonlinear processes may in turn generate ton acoustic wave curbulence with fon acoustlc waves characterized by a broad sperctrum. All these processes can take place on a time scale of a fow picoseconds, which is shorter than the time necessary for the SBS gain factor to be larger than unity. Thus the coupling of plasma waves to fon acoustic waves could represent un eflicient mexhanism for giving rise to an anomalous level of lon acoustic wave nolse from which SBSS can then develop. It would consequently be of interest for future short pulse experiments to Investigate the SRS/SBS correlation. In particular, future experiments performed in plasmas with a lower density in order to suppress SRS would make possible to examine whether the SBSS refectivity becomes the 'Thomson scattering reflectlvity TS, as theoretically predicted. The internetion betweon these two instabilitles have been observed in a number of experiments under different plasma and laser conditions |20|.

The utllization of short laser pulses with ps duration offers the possibility of studying the growth and evolution of parametric instabilities more detall than what has been done until now. Ideally one would like to vary the pulse duration so as to control the number of e-folding in the growth. Varying the pulse duration and intensity allows the separate control study of issues related to the growth and nolse levels and from nonlinear issues leading to saturation. Using pulses with duration much shorter than 1 ps (c.g. 100 is) would nllow the study of the growth of SRS as woll, an instabillty with faster growth than S13S. Shorter pulses will also permit the study of SBS at much higher laser intensities and still avoid saturation. Further improvements in coming experiments could include the 


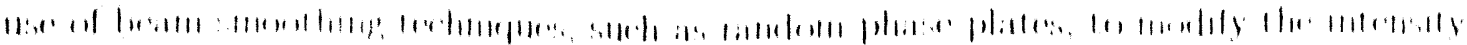

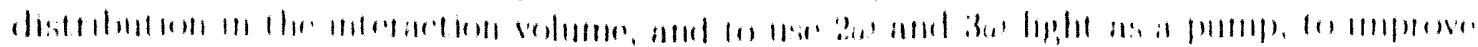

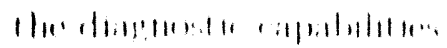

\section{A(:IINOWI,HI)(IMIR:NT}

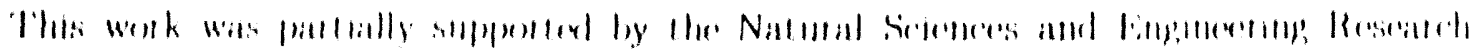

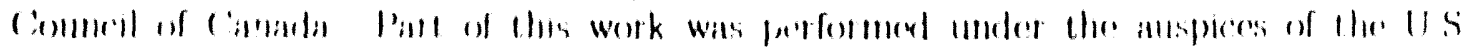

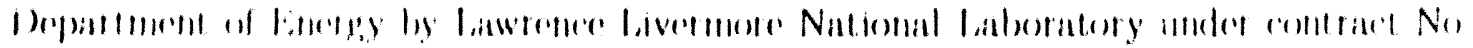

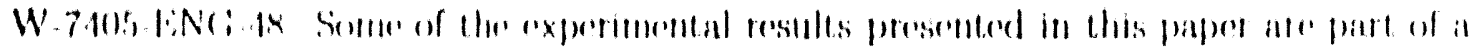

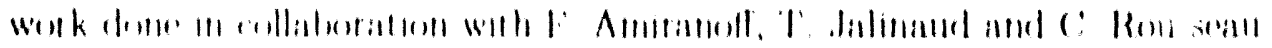

\section{Reforencess}

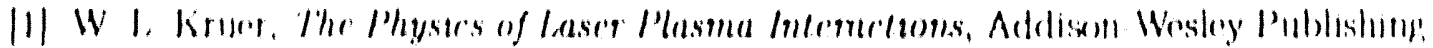
(impially, lor, 1!ns

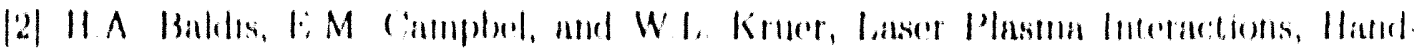

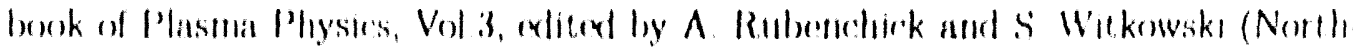
Holland, Amsiterdam, 1992)

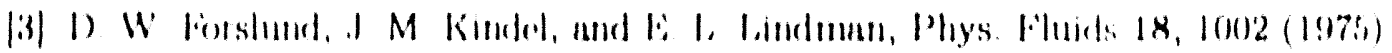

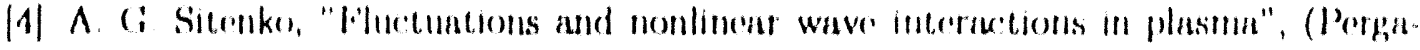
mon, New York, 19:2), (hap. 4, p. 126

|5| II. A. Baldis, D. M Villeneuve, B. Lalontaine, ( . D. Linrighit, C. Labaunc, s. Baton, Phe Moumix, 1). Pesme, M. Casanova and W. Rozmus, "Stlmulated Brillonin seattering, in picosecond time scales : experiments and modeling", submitterl to Phys Fluids B

(6) Ph. Mounaix, 1). Pesme, M. Casanova and W. Rozmus, "Space and lime behavion of parametric instabilities for a finite pump wave duration in a bounded plasma", submituer to Phys Fluids B.

|7| B. A. Williams, R. R. McGowan, ICF Quarterly Report 1,147 (1991), Lawrence Livernore National Laboratory, Livermore CA, UCl.R-LR-105821-91-4.

|8| D. F. DußBois (Private communication).

|9| H. A. Rose, D. F. DußBois, B. Bexzerides, Phys. Rev. Lett.58, 2547 (1987).

|10| W. Rozmus, R.P. Sharma, J.C. Samson, W.'Tigho, Phys. Fluids 30, 2181 (1987)

111| G. Bonnand, D. Pesme, R. Pollat, Phys. Fluids B 2, 1618 (1990).

(12) T. Kolber, W. Rozmus, V. T. Tikhonchuk, Phys. Pluids B 5, 138 (1993)

|13| B. Bezzerides, D. F. DulBois, H. A. Rose, "Saturation of stimulated Raman scuttering by the excitation of strong Langmuir turbulence", submitted to Phys. Rev. lett.

|11| T. Kolber, W. Rommus, V. 'Tikhonchuk, Ph. Mounaix, and D. Pesme, "laser light scattering from fluctuations onhanced by stimulated Raman scattering", Phys. Rev. Jett. (in press, 1993). 


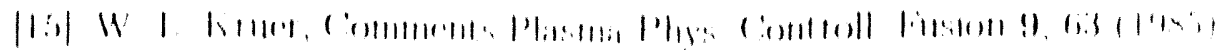

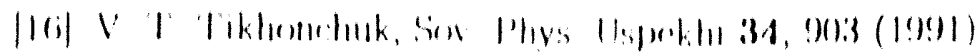

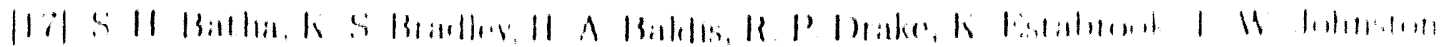

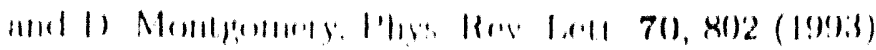

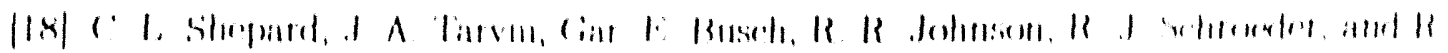

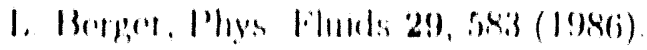

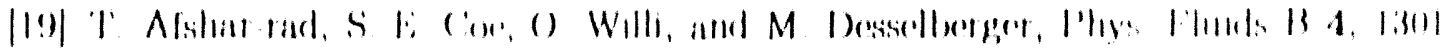
(19!92)

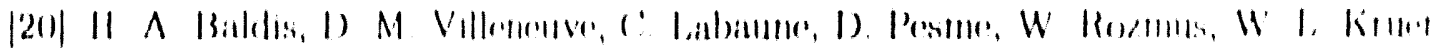

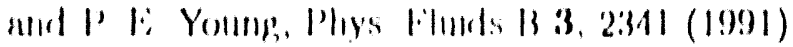

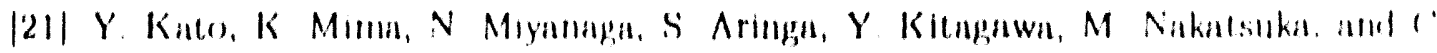

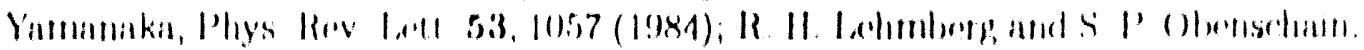

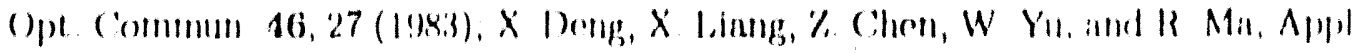
()ptics $25,377(1986)$

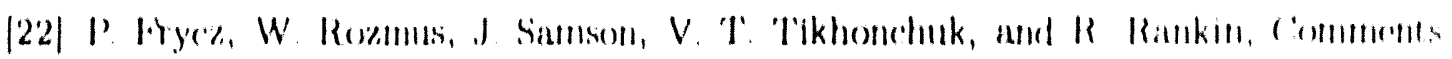
Plasma Phys. Comtroll Rusion 15, 19 (1992).

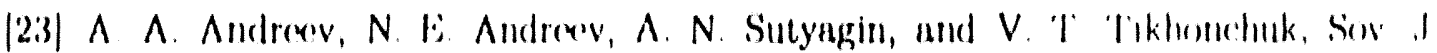
Plasma Plyys 15, 546 (1989)

|21| R.W. Short, and F.M Lipporlann, Pys. Rev. Lett. 68, 3307 (19912)

|25) H. A. Rose and D.F. Dubols, Phys. Fluids B 41394 (1992)

|26| M. R. Amin, C. F. Capjuck, P. lityez, W. Rozmus, und V. 'T. 'Tikhondhuk, "I'wo dimonsional studies of stimulated Brillouin scattering, filamentation and self-focussing instabilities of lawer light in plasmas", Phys. Fluids, submittical for publication (1993).

|27|' A. J. Schmitt, Phys. Flujds 31, 3079 (1988).

|28| W. Rozmus, M. Casanova, D. Pesme, A. Heron, and J.-C. Adam, Plyss Fluids Bs 4. $570(1992)$.

|29| R. H. Lehmberg, Phys. Rev. Leth. A1, 863 (1978).

|30| B. Ya. Zel'dovich, N. F. Pilipetsky, and V. V. Shkunov, Principles of Phase Cionjugation, Springer Verlag, Berlin (1985) |Springer Series in Optical Scionces, Vol 42|.

|31| C. J. Mckinstric and M. V. Goldman, J. Opt. Soc. Am. 9, 1778 (1992).

|32| N. E. Andreev and L. M. Gorbunov, JETP I Aett. 56, 144 (1992)

|33| R. D. Jones, W. C. Mead, S. W. Coggeshall, C. II. Aldrich, I I. Norton, (; 1) Pollak, and J. M. Wallace, Phys. Fluids 31, 1249 (1988).

|34| F. Amiranoff, C. Labaune, G. Matthieussent, B. Mabille, P. Mora, I). Pesme, M Haguenauer, LULI Annual report, 50 (1986)

|35| D. Bruneau, A.M. Tournade and E. Fabre, Appl. Opt. 24, 3740 (1985) 


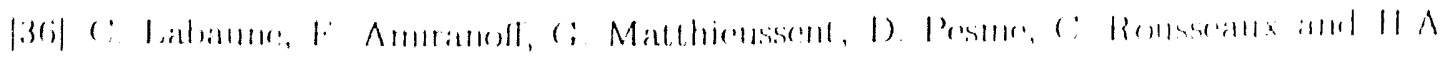
Baklis Rev lhys Appl. 23, 1505) (1988).

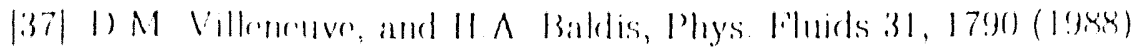

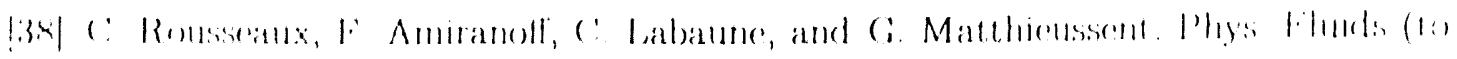
lar puhlish. I!3!3)

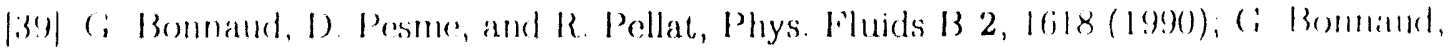
1) Pesme, and !r. Pellat, ibici 4, 423 (19:32).

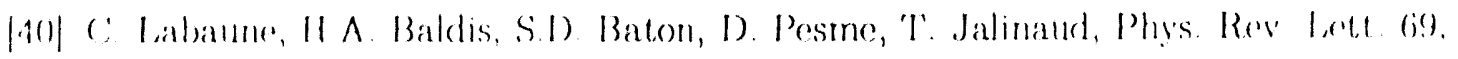
$245,(1992)$

411 .I A Iloikkinen and S.A. Kartumen, Phys. Fluids 29, 1291 (1986)

|42! (: I abanne, S.1) Batu, 'T, Jalinand, H.A. Baldis, D. Pesme, Phys. Pluids. B4, $2224(1992)$

(43) CS. Liu and V.K. Thipathi, Phys. Fluids 29, 4188 (1986). R.W. Short, W. Soka and R Bahr, Phys. Fluids 30, 3245 (1987). H.C. Barr, T.J Boyd and C. A Coutls, Phys. Rov Ioll, 56, $2256(1986)$

|41| PK. Kaw, G. Schmidt, and T. Wilcox, Phys. Fluids 16, 1522 (1973).

|45| E. Ei Epperlein, Phys.Rev. Lett. 65,2145 (1990).

|46| C.S. Liu, M.N. Rosenbluth, and R.B. White, Phys. Fluids 19, 1211 (1974).

|47| (:.J. Walsh, D.M. Villeneuve, and H.A. Baldis, Phys. Rev. Lett. 53, 1445 (1984).

|48| II.A. Baldis, R.P. Drake, W.L. Kruer, K.G. Estabrook, E.A. Williams, and T. W. Johnston, Phys. Rev. Iett. 62, 2829 (19899).

|49| C.13. Darrow, C. Cloverdale, M.D. Perry, W.B. Mori, C. Clayton, K. Marsh, and ('. Joshi, Phys Rev. Lett. 69, 442 (1992).

150|: S.C. Wilks, W.L. Kruer, A.B. Langdon, P. Amendt, D.C. Eder, and C.J. Keane, Proc. SPIE "Short-Pulse High-Intensity Lasers and Applications" ,1413, 131 (1991). 

(2)

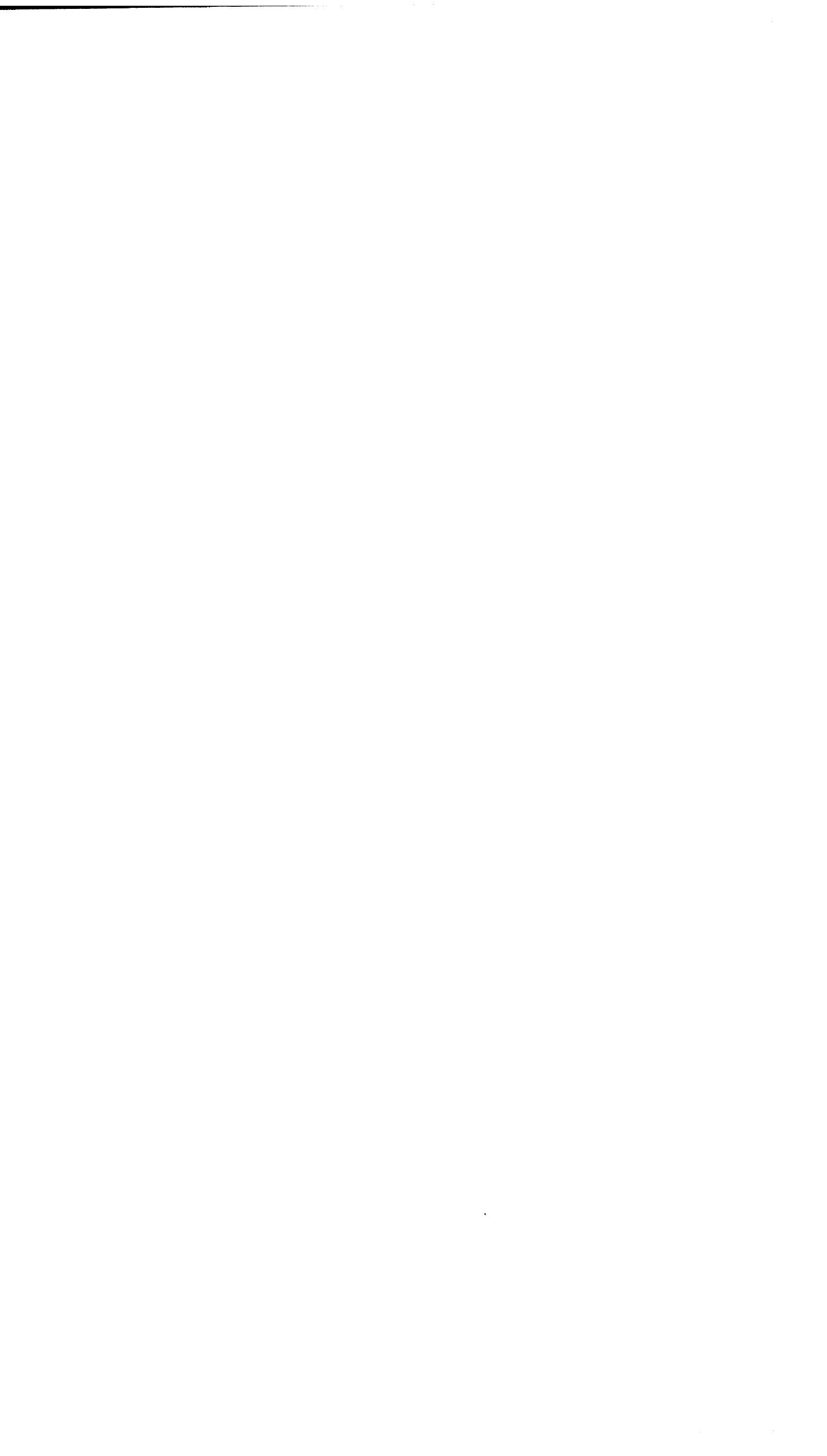

